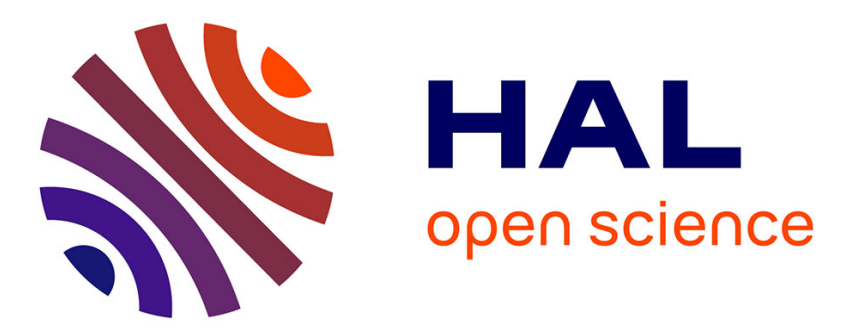

\title{
Numerical study of non-isothermal adsorption of Naphthalene in supercritical CO2: behavior near critical point
}

Manel Wannassi, Isabelle Raspo

\section{- To cite this version:}

Manel Wannassi, Isabelle Raspo. Numerical study of non-isothermal adsorption of Naphthalene in supercritical CO2: behavior near critical point. Journal of Supercritical Fluids, 2016, 117, pp.203-218. 10.1016/j.supflu.2016.06.020 . hal-01369830

\section{HAL Id: hal-01369830 \\ https://hal.science/hal-01369830}

Submitted on 6 Apr 2017

HAL is a multi-disciplinary open access archive for the deposit and dissemination of scientific research documents, whether they are published or not. The documents may come from teaching and research institutions in France or abroad, or from public or private research centers.
L'archive ouverte pluridisciplinaire HAL, est destinée au dépôt et à la diffusion de documents scientifiques de niveau recherche, publiés ou non, émanant des établissements d'enseignement et de recherche français ou étrangers, des laboratoires publics ou privés. 


\section{Accepted Manuscript}

Title: Numerical study of non-isothermal adsorption of

Naphthalene in supercritical $\mathrm{CO}_{2}$ : behavior near critical point

Author: Manel Wannassi Isabelle Raspo

PII: $\quad$ S0896-8446(16)30196-6

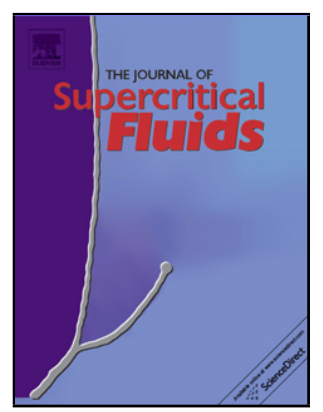

DOI: $\quad$ http://dx.doi.org/doi:10.1016/j.supflu.2016.06.020

Reference: $\quad$ SUPFLU 3697

To appear in: $\quad$ J. of Supercritical Fluids

Received date: $\quad$ 23-3-2016

Revised date: 29-6-2016

Accepted date: $\quad 30-6-2016$

Please cite this article as: M. Wannassi, I. Raspo, Numerical study of non-isothermal adsorption of Naphthalene in supercritical $\mathrm{CO}_{2}$ : behavior near critical point, The Journal of Supercritical Fluids (2016), http://dx.doi.org/10.1016/j.supflu.2016.06.020

This is a PDF file of an unedited manuscript that has been accepted for publication. As a service to our customers we are providing this early version of the manuscript. The manuscript will undergo copyediting, typesetting, and review of the resulting proof before it is published in its final form. Please note that during the production process errors may be discovered which could affect the content, and all legal disclaimers that apply to the journal pertain. 


\section{Highlights}

4

5

6

7

8

9

10

11

12

13

14

15

16

17

18

19

20

21

22

23

\section{(1)}

2

- Non-isothermal adsorption in near-critical binary mixtures was investigated by numerical simulations.

- The adsorption behavior near solvent's critical point has been analyzed.

- The effect of divergent properties and the piston effect were highlighted.

- A strong dependence to temperature and pressure variations in the vicinity of the critical point was depicted.

13




\title{
Numerical study of non-isothermal adsorption of Naphthalene in supercritical $\mathrm{CO}_{2}$ : behavior near critical point
}

\author{
Manel Wannassi*, Isabelle Raspo
}

Aix Marseille Univ, CNRS, Centrale Marseille, M2P2, Marseille, France

\begin{abstract}
In this study, adsorption in a model binary mixture is investigated near the critical point in a side-heated cavity. The diverging behavior of the equilibrium constant and the Piston effect are taken into account and their influence on the adsorption process is pointed to. The modeling is based on numerical integration of the differential equations, considering the Navier-Stokes equations coupled with the energy and mass diffusion balances. By means of this model, the temperature, density and adsorbed concentration profiles are drawn at different times. Some fundamental concepts about the system's response to the heating are illustrated. The results reveal that the adsorption process is influenced by the combined effect of several parameters, such as the gravity and the proximity to the critical point. In particular, the adsorbed amount exhibits a reversed dependency on the wall heating very close to the critical point, which confirms the complexity of such a process in binary systems near critical conditions.
\end{abstract}

Keywords: Supercritical fluids; Adsorption; Piston effect; Numerical analysis

\section{Introduction}

The supercritical state was first reported in 1822 by Baron Gagniard de la Tour [1], but only one hundred years later, supercritical techniques have received increased attention and have been used in analytical and on an industrial scale. This state is achieved when the temperature and the pressure of a substance is set over their critical values. So the properties 
of a supercritical fluid range between those of a liquid and a gas and the distinction between the liquid and the gas phases is not possible. Some of the properties of a supercritical fluid are more liquid-like, whereas others are more gas-like.

Moreover, very close to the critical point, some properties diverge and others tend to zero. In fact, a small raise in pressure remarkably increases the fluid density and this effect diminishes with increasing distance from the critical point. On the other hand, a supercritical fluid has a higher diffusion coefficient and lower viscosity and surface tension than a liquid solvent, which leads to a more favorable mass transfer. Supercritical fluids exhibit very interesting qualities with regard to their physicochemical properties as well as ecology and economy. They are used as an alternative to organic liquid solvents in several applications such as cleanings [2-4]. Adsorption technologies using supercritical fluids have been also focused due to their potential applications including analytical extractions, activated carbon regeneration and soil remediation. Several studies have investigated the supercritical adsorption characteristics of many systems [5-12]. When adsorption is concerned, thermodynamic and kinetic aspects should be involved to know more details about its performance and mechanisms.

In the framework of isothermal supercritical adsorption, there have been numerous publications in literature dealing with the modeling of adsorption equilibrium using the most common adsorption isotherm models, i.e. the Langmuir, the Freundlich and the RedlichPeterson models [6-7, 13-16]. All the experimental conditions used correspond to thermodynamic states relatively beyond the critical point because the adsorption equilibrium is influenced by the system temperature, pressure and by the supercritical fluid properties in the vicinity of the critical point. In contrast, supercritical adsorption systems close to the solvent's critical point have received much less explicit attention in the open literature. The experimental studies in this area are scarce. A thermodynamic analysis of near critical binary 
mixtures was established by Afrane and Chimowitz [17]. The authors studied the adsorption thermodynamics of dilute solutes adsorbing from high pressure supercritical fluid using the Henry's law. However, set under high pressures, the results showed an extremely weak dependence to pressure and to the composition of the supercritical solvent phase. In chromatography, the proximity to the critical point was early reviewed by Van Wasen et al. [20]. The authors pointed out the unusual behavior of equilibrium partition coefficients in the near-critical region. Many other works also showed interesting features of data in this region [21-22]; in particular, papers by Schmitz et al. [23] and Klesper and Schmitz [24] provided striking evidence of the highly nonlinear behavior of equilibrium coefficients with respect to pressure and temperature variations, as the critical point of the fluid phase is approached. We believe that an adequate explanation of the thermodynamic basis of these phenomena in adsorption process taking into account both temperature and pressure effects is necessary. And it is also important to show the influence of the divergent character of thermodynamic properties and transport coefficients in near-critical systems on adsorption system behavior.

This is precisely the aim of this paper. For this purpose, adsorption of a model solute from supercritical $\mathrm{CO}_{2}$ was investigated in a small side-heated cavity by means of $2 \mathrm{D}$ numerical simulations. Naphthalene was chosen as a model solute because its phase equilibria with $\mathrm{CO}_{2}$ has been thoroughly studied [18-19]. There are extensive data available for this system that have been confirmed. The first section of the paper is devoted to the mathematical modeling of the problem and the numerical method used for the simulations. The modeling of the adsorption reaction at the solid boundaries is exposed in details. Then, the effect of the mass fraction and the proximity to the critical point are discussed for wide temperature and pressure conditions. The results show a strong dependence to temperature and pressure variations when the critical point is approached. We ended up with the effect of Damköhler number on the adsorbed mass fraction. 
2. Mathematical modeling

\subsection{Problem under investigation}

The problem we consider is that of a dilute solute (Naphthalene in this case, named species 2) in supercritical $\mathrm{CO}_{2}$ (named species 1). The physical properties of each pure compound are given in Table 1 . The Naphthalene- $\mathrm{CO}_{2}$ mixture is enclosed in a square cavity of height $H=1 \mathrm{~mm}$ and subjected to the earth gravitational field g. The cavity vertical walls are made of activated carbon (see Fig. 1). The activated carbon was chosen as a model adsorbent for this problem allowing as considering an adsorption reaction at the solid-fluid interface. Here, we emphasize that the chosen mixture as the adsorbent material is only generic since the aim of this study is to qualitatively investigate the influence of the proximity to the critical point on an adsorption reaction. Initially, the fluid is considered in thermodynamic equilibrium at a constant temperature $T_{i}$ slightly above the mixture critical temperature $T_{c m}=307.65 \mathrm{~K}$ such that $T_{i}=(1+\varepsilon) T_{c m}$, where $\varepsilon$ defines the dimensionless proximity to the critical point $(\varepsilon<<1)$, and the density is equal to the mixture critical density $\rho_{c m}=470 \mathrm{~kg} \cdot \mathrm{m}^{-3}$. The critical properties, $T_{c m}$ and $\rho_{c m}$ correspond to the LCEP ("Lower Critical EndPoint") of the mixture and are slightly above the critical point of $\mathrm{CO}_{2}$ $\left(T_{c 1}=304.21 \mathrm{~K}, \rho_{c 1}=467.8 \mathrm{~kg} \cdot \mathrm{m}^{-3}\right)$. A weak gradually heating is then applied at the solid plate $(\mathrm{x}=0)$. The hot temperature is noted $T_{h}=T_{i}+\delta T$ where $\delta \mathrm{T}$ is about hundreds $\mathrm{mK}$, while condition was applied to the non-reactive walls. 


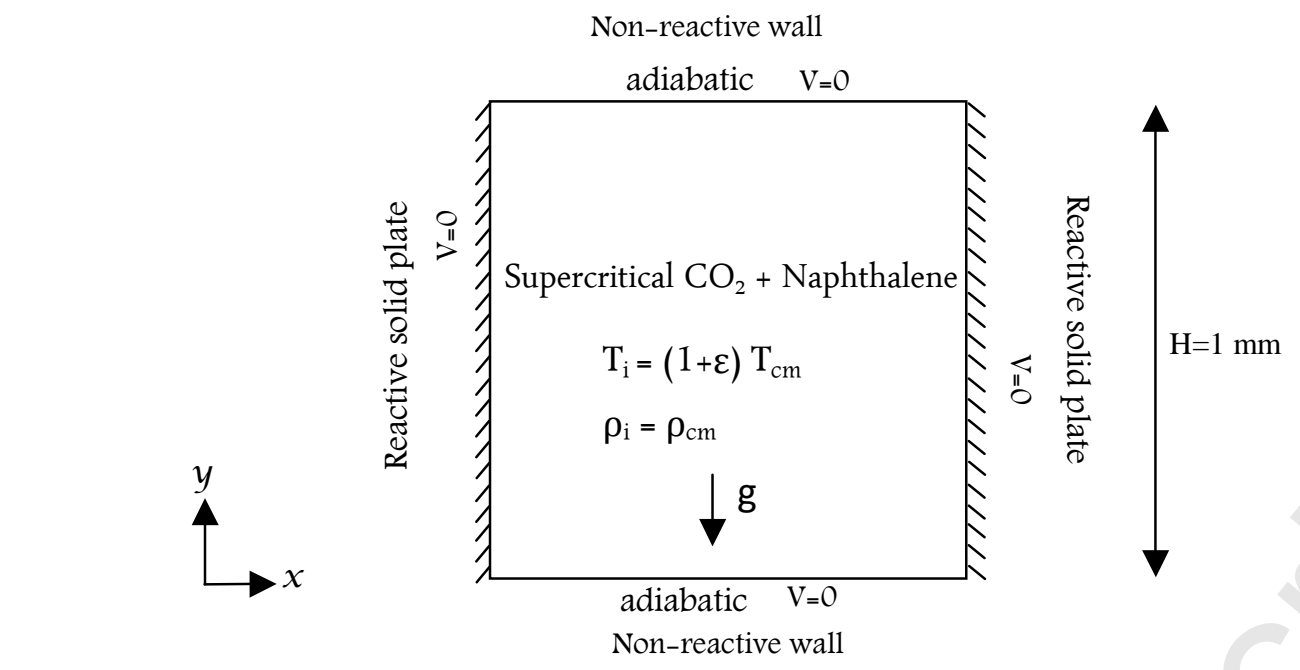

135

Fig. 1. Physical configuration

Table 1

Pure component properties

\begin{tabular}{|c|c|c|c|c|c|c|c|}
\hline & $\mathbf{T}_{\mathrm{c}}(\mathbf{K})$ & $\rho_{c}\left(\right.$ kg.m $\left.{ }^{-3}\right)$ & $\mathbf{P}_{\mathrm{c}}(\mathrm{bar})$ & $\mathrm{M}\left(\mathrm{kg} \cdot \mathrm{mol}^{-1}\right)$ & $\omega$ & $v_{b}\left(\mathrm{~cm}^{3} \mathrm{~mol}^{-1}\right)$ & Ea $\left(\mathrm{J} \mathrm{mol}^{-1}\right)$ \\
\hline $\mathrm{CO}_{2}(1)$ & 304.21 & 467.8 & 73.8 & $4.40110^{-2}$ & 0.225 & - & - \\
\hline Naphthalene (2) & 748.40 & 314.9 & 40.5 & $1.28210^{-1}$ & 0.302 & 155 & 101.4 \\
\hline
\end{tabular}

137

138

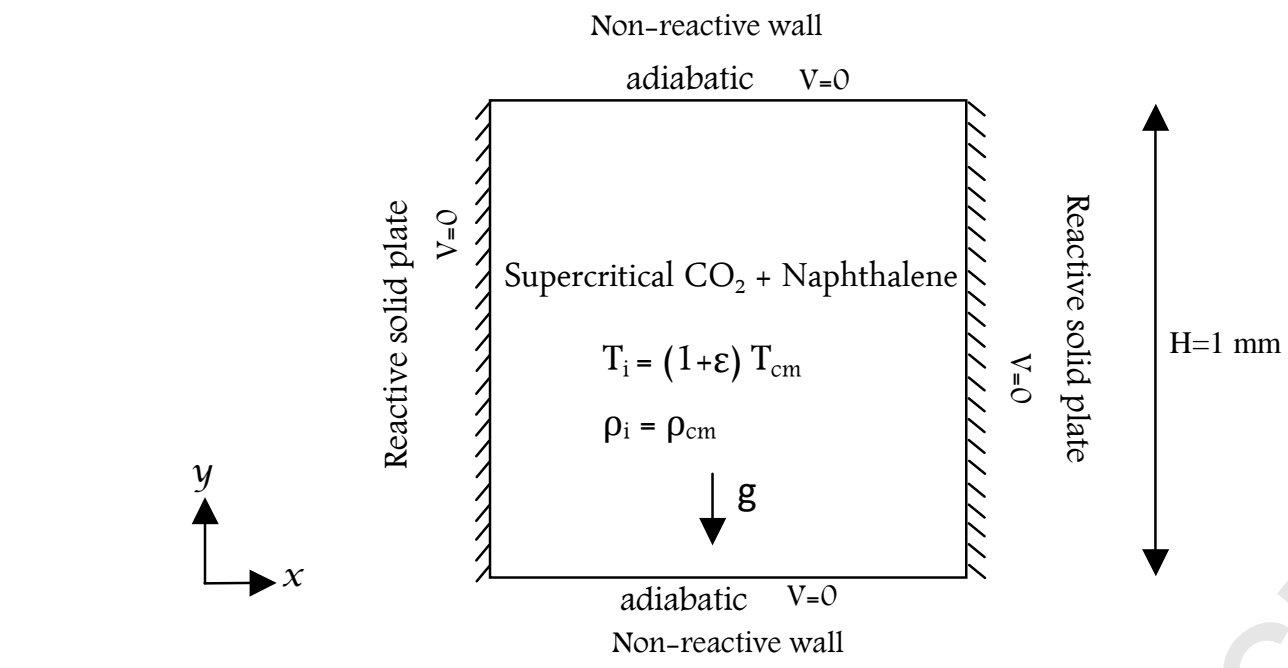

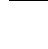


Table 2

Initial parameters

\begin{tabular}{|c|c|c|c|c|c|}
\hline $\mathbf{T}_{\mathbf{i}}(\mathbf{K})$ & $\rho_{\mathrm{i}}\left(\mathrm{kg} \cdot \mathrm{m}^{-3}\right)$ & $\lambda_{i}\left(W \cdot m^{-1} \cdot K^{-1}\right)$ & $\mathrm{Cv}_{\mathrm{i}}\left(\mathrm{J} \mathrm{kg}^{-1} \cdot \mathrm{K}^{-1}\right)$ & $\mu_{\mathrm{i}}($ Pa.s) & $\left(D_{21}\right)_{i}\left(m^{2} \cdot s^{-1}\right)$ \\
\hline 307.75 & 470 & 0.098332532 & 1325.839 & $3.33828 \times 10^{-5}$ & $2.19525 \times 10^{-8}$ \\
\hline 308.15 & 470 & 0.096196327 & 1306.27 & $3.34016 \times 10^{-5}$ & $2.19686 \times 10^{-8}$ \\
\hline 309.15 & 470 & 0.091343811 & 1269.32 & $3.34485 \times 10^{-5}$ & $2.20090 \times 10^{-8}$ \\
\hline 311.15 & 470 & 0.083368892 & 1214.86 & $3.35423 \times 10^{-5}$ & $2.20895 \times 10^{-8}$ \\
\hline 318.15 & 470 & 0.066814297 & 1074.57 & $3.38700 \times 10^{-5}$ & $2.23679 \times 10^{-8}$ \\
\hline
\end{tabular}

total pressure. In [38], a modification of the Low Mach number Approximation was proposed to account for the strong stratification of fluids near the critical point. We tested this modification and we noted that, for the present problem, the results obtained with and without the modification were the same. Therefore, the original approximation [25] was used for the simulations reported in this paper.
155

The dimensionless formulation was obtained using $T_{c m}$ as characteristic temperature, $\rho_{i}$ as characteristic density, $\rho_{i}\left(R / M_{1}\right) T_{c m}$ (with $\mathrm{R}$ is the perfect gas constant $\left(\mathrm{R}=8.3145 \mathrm{~J} \mathrm{~mol}^{-1} \mathrm{~K}^{-}\right.$ $\left.{ }^{1}\right)$ ) as characteristic pressure, $H$ as characteristic length, the time scale of the piston effect as characteristic time, $t_{P E}=\frac{t_{d}}{\left(\gamma_{m}-1\right)^{2}}$, where $t_{d}$ is the characteristic time of thermal diffusion, $\gamma_{m}$ the capacity ratio of the mixture (see Appendix A) and $H / t_{P E}$ was taken as the characteristic velocity. The transport properties such as the dynamic viscosity $\mu$, the isochoric specific heat capacity $C_{V}$, the thermal conductivity $\lambda$ and the diffusion coefficient $D_{21}$ were dimensionless, relative to their respective initial values $\left(\mu_{i}, \lambda_{i}, C_{v i},\left(D_{21}\right)_{i}\right)$. Thus, the governing equations in a dimensionless form are:

$$
\frac{\partial \rho}{\partial t}+\nabla \cdot(\rho \mathbf{V})=0
$$

$\rho \frac{\partial \mathbf{V}}{\partial t}+\rho \mathbf{V} \cdot \nabla \mathbf{V}=-\nabla P_{d y n}+\frac{1}{\operatorname{Re}} \Delta \mathbf{V}+\frac{1}{3 \operatorname{Re}} \nabla(\nabla \cdot \mathbf{V})+\frac{1}{F r} \rho$ 
$\rho \frac{\partial T}{\partial t}+\rho \mathbf{V} . \nabla T=-\frac{C_{v 0}}{C_{v i}}\left(\gamma_{0}-1\right)\left[P_{t h}-T\left(\frac{\partial P_{t h}}{\partial T}\right)_{\rho, w}\right](\nabla . \mathbf{V})$

$+\frac{\gamma}{\operatorname{RePr}} \nabla \cdot\left(\lambda^{*} \nabla T\right)-\left[\left(\bar{U}_{2}^{*}-\bar{U}_{1}^{*}\right)+\frac{C_{v 0}}{C_{v i}}\left(\gamma_{0}-1\right)\left(P_{t h}-T\left(\frac{\partial P_{t h}}{\partial T}\right)_{\rho, w}\right)\left(\bar{V}_{2}^{*}-\bar{V}_{1}^{*}\right)\right] \times$

$\frac{1}{(\gamma-1)^{2} L e \theta(w)} \nabla \cdot\left(\rho D_{21}^{*} \nabla w\right)$

$\rho \frac{\partial w}{\partial t}+\rho \mathbf{V} \cdot \nabla w=\frac{1}{(\gamma-1)^{2} L e} \nabla \cdot\left(\rho D_{21}^{*} \nabla w\right)$

$169 V$ is the velocity of components $u$ and $v$ in the $x$-and $y$-directions respectively, $w$ is the mass

170 fraction, $\gamma$ is the ratio of the isobaric and isochoric specific heats calculated from the

171 equation of state (see Appendix A) with $\gamma_{0}$ and $C_{v 0}$ corresponding to the values for a perfect

172 gas $\left(\gamma_{0}=1.3, C_{v 0}=3 R / M_{1}\right)$. The value of $C_{v i}$ for the initial state was taken from the NIST

173 (see Table 2). The dimensionless numbers are respectively, the Mach number $M a$, the

174 Reynolds number Re, the Froude number Fr, the Prandtl number Pr and the Lewis number Le

175 and are defined as:

$176 \quad M a=\frac{V_{P E}}{c_{0}}, \quad \operatorname{Re}=\frac{\rho_{i} V_{P E} H}{\mu_{i}}, \quad F r=\frac{V_{P E}^{2}}{g H}, \quad \operatorname{Pr}=\frac{\mu_{i} \gamma C_{v i}}{\lambda_{i}}, \quad L e=\frac{\lambda_{i}}{\rho_{i} \gamma C_{v i}\left(D_{21}\right)_{i}}$

177 where $c_{0}=\sqrt{\gamma_{0}\left(R / M_{1}\right) T_{c m}}$ is the sound speed and $V_{P E}=H / t_{P E}$ is the characteristic velocity 178 of the piston effect.

Table 3

Characteristic times (piston effect $t_{\mathrm{PE}}$, thermal diffusion $\mathrm{t}_{\mathrm{d}}$, mass diffusion $\mathrm{t}_{\mathrm{Md}}$, adsorption $\mathrm{t}_{\mathrm{ad}}$ )

\begin{tabular}{lllll}
\hline $\mathbf{T}_{\mathbf{i}}(\mathbf{K})$ & $\mathbf{t}_{\mathbf{P E}}(\mathbf{s})$ & $\mathbf{t}_{\mathbf{d}}(\mathbf{s})$ & $\mathbf{t}_{\mathbf{M d}}(\mathbf{s})$ & $\mathbf{t}_{\mathbf{a d}}(\mathbf{s})$ \\
\hline 307.75 & 0.1999 & 115.6989 & 45.5530 & $45.5530 \times 10^{5}$ \\
308.15 & 0.2367 & 106.9022 & 45.5194 & $45.5194 \times 10^{5}$ \\
309.15 & 0.3333 & 91.1253 & 45.4359 & $45.4359 \times 10^{5}$ \\
311.15 & 0.5404 & 73.0746 & 45.2704 & $45.2704 \times 10^{5}$ \\
318.15 & 1.2621 & 49.2332 & 44.7069 & $44.7069 \times 10^{5}$ \\
\hline
\end{tabular}


In Eq. (3), $\bar{U}_{k}^{*}$ and $\bar{V}_{k}^{*}$ are respectively the dimensionless partial molar internal energy and

181 partial molar volume expressed as follow:

$182 \bar{U}_{k}^{*}=\bar{U}_{k} /\left(M_{2} C_{v i} T_{c m}\right)$ and $\bar{V}_{k}^{*}=\bar{V}_{k} /\left(M_{2} / \rho_{i}\right)$ for $\mathrm{k}=1,2$.

183 The expressions of $\bar{U}_{k}$ and $\bar{V}_{k}$ calculated using the Peng Robinson equation of state are

184 given in Appendix B.

185 The following relationship is used for $\theta(w)$ :

186

$\theta(w)=1-\left(1-\frac{M_{1}}{M_{2}}\right) w$

187 with $M_{1}$ and $M_{2}\left(\mathrm{~kg} \mathrm{~mol}^{-1}\right)$ are respectively, the molecular weight of $\mathrm{CO}_{2}$ and Naphthalene 188 (see Table 1).

189 The superscript $(*)$ refers to dimensionless parameters.

190 For thermal conductivity $\lambda\left(\mathrm{W} \cdot \mathrm{m}^{-1} \cdot \mathrm{K}^{-1}\right)$, the following correlation is used [26]:

191

$\lambda(T, \rho)=\lambda_{0}(T)+\lambda_{e}(\rho)+\Delta \lambda_{c}(T, \rho)$,

192 The first term $\lambda_{0}(T)$ corresponds to the limit of small densities and is expressed as follow:

$\lambda_{0}(T)=-7.6683 \times 10^{-3}+8.0321 \times 10^{-5} T$

194 The second term $\lambda_{e}(\rho)$ is the excess property and is expressed as follow:

195

$\lambda_{e}(\rho)=3.0990 \times 10^{-5} \rho+5.5782 \times 10^{-8} \rho^{2}+2.5990 \times 10^{-17} \rho^{5}$,

196 And the third term is the critical enhancement:

197
$\Delta \lambda_{c}(T, \rho)=\left(\frac{1.6735}{T-291.4686}-0.2774+7.4216 \times 10^{-4} T\right) \times \exp \left(-C^{2}\left(\rho-\rho_{c 1}\right)^{2}\right)$ 
198 with $\begin{cases}C=6.7112 \times 10^{-3} & \text { if } \rho<\rho_{c 1} \\ C=6.9818 \times 10^{-3} & \text { if } \rho>\rho_{c 1}\end{cases}$

199 The binary mass diffusion coefficient, $D_{21}\left(\mathrm{~m}^{2} \mathrm{~s}^{-1}\right)$, is calculated with the Wilke-Chang 200 equation [27]:

201

$$
D_{21}=7.4 \times 10^{-15} \frac{T \sqrt{\Phi 10^{3} M_{1}}}{\mu \vartheta_{b 2}^{0.6}},
$$

202 with $\vartheta_{b 2}\left(\mathrm{~cm}^{3} \mathrm{~mol}^{-1}\right)$ the molar volume of Naphthalene at boiling point, $\Phi$ the association 203 factor $\left(\Phi=1\right.$ for $\left.\mathrm{CO}_{2}\right)$.

204 The thermodynamic state of the mixture is described by the Ping-Robinson equation of state 205 in the framework of the one-fluid theory. We can then compute the thermodynamic pressure 206 as follow:

207

$$
P_{t h}=\frac{T \rho \theta(w)}{1-b^{*}(w) \rho / \theta(w)}-\frac{a^{*}(T, w) \rho^{2}}{1+2 b^{*}(w) \rho / \theta(w)-b^{*}(w)^{2} \rho^{2} / \theta(w)^{2}}
$$

208 Where:

209 $a^{*}(T, w)=a_{1}^{*}(T)(1-w)^{2}+2 a^{*}{ }_{12}(T) w(1-w)+a_{2}^{*}(T) w^{2}$,

$$
b^{*}(w)=b_{1}^{*}(1-w)^{2}+2 b_{12}^{*} w(1-w)+b_{2}^{*} w^{2},
$$

$$
a_{1}^{*}(T)=1.487422 \frac{T_{c 1}}{T_{c m}} \frac{\rho_{i}}{\rho_{c 1}}\left[1+\beta_{1}\left(1-\sqrt{T\left(T_{c m} / T_{c 1}\right)}\right)\right]^{2}
$$

$$
a_{2}^{*}(T)=1.487422 \frac{M_{1}}{M_{2}} \frac{T_{c 2}}{T_{c m}} \frac{\rho_{i}}{\rho_{c 2}}\left[1+\beta_{2}\left(1-\sqrt{T\left(T_{c m} / T_{c 2}\right)}\right)\right]^{2}
$$

$$
b_{j}^{*}=0.253076\left(\frac{\rho_{i}}{\rho_{c j}}\right) \text { with } \mathrm{j}=(1,2)
$$


$a^{*}{ }_{12}(T)=\sqrt{a_{1}^{*}(T) a_{2}^{*}(T)}\left(1-k_{12}\right)$

$215 \quad b_{12}^{*}=\frac{1}{2}\left(b^{*}{ }_{1} \frac{M_{1}}{M_{2}}+b^{*}{ }_{2}\right)\left(1-l_{12}\right)$,

216

$\beta_{j}=0.37464+1.54226 \omega_{j}-0.26992 \omega_{j}^{2} \quad(\mathrm{j}=1,2)$

217 with $\omega$ the acentric factor (Table 1). The binary interaction parameters $k_{12}$ and $l_{12}$ are

218 determined so as to minimize the error between the calculated and experimental solubility 219 data. These two parameters are temperature dependent and they are obtained through these 220 formulae [28]:

221

$k_{12}=k_{12}^{\prime}+k_{12}^{\prime \prime}\left(\frac{308.15}{T}-1\right)$

222

$l_{12}=l_{12}^{\prime}+l_{12}^{\prime \prime}\left(\frac{308.15}{T}-1\right)$

The values of the binary interaction parameters predicted by a least square method are then:

$k_{12}^{\prime}=0.0395, k_{12}^{\prime \prime}=0.0114, l_{12}^{\prime}=-0.1136$ and $l_{12}^{\prime \prime}=-0.3103$.

As it can be noted, the equations (1) - (5) are coupled for a given time step. This coupling can be reduced by using an explicit scheme to evaluate the convective terms in Eq. (3). But the energy source term involving $\nabla . V$ must be implicitly evaluated because it accounts for the piston effect, namely the thermoacoustic effect responsible for fast heat transfer near the liquid-gas critical point. So in order to decouple the energy equation (Eq. (3)) and the Navier- 
231 Stokes equations (Eqs. (1)- (2)), the velocity divergence, must be calculated using only the

232

233

234

235

236

237

$\frac{d T}{d t}=\frac{-\left(\frac{\partial F}{\partial \rho}\right)_{T, w} \frac{d \rho}{d t}+\frac{d P_{t h}}{d t}-\left(\frac{\partial F}{\partial w}\right)_{T, \rho} \frac{d w}{d t}}{\left(\frac{\partial F}{\partial T}\right)_{\rho, w}}$

238 Moreover, the continuity equation (1) can also be written in the following form:

239

$240 \quad \frac{d \rho}{d t}=-\rho(\nabla . V)$

Then, inserting Eq. (6) in the energy equation Eq. (3) using Eq. (7) for the computation of $243 d \rho / d t$ and Eq. (4) for the computation of $d \mathrm{w} / d t$, lead finally to the following expression for the velocity divergence:

$\nabla . \mathbf{V}=\left\{\begin{array}{l}\rho \frac{d P_{t h}}{d t}-\frac{\gamma}{\operatorname{Re} \operatorname{Pr}}\left(\frac{\partial F}{\partial T}\right)_{\rho, w} \nabla \cdot\left(\lambda^{*} \nabla T\right)-\left[\left(\frac{\partial F}{\partial w}\right)_{T, \rho}-\frac{1}{\theta(w)} A\left(\bar{U}^{*}, \bar{V}^{*}\right)\left(\frac{\partial F}{\partial T}\right)_{\rho, w}\right] \\ \times\left[\frac{1}{(\gamma-1)^{2} L e} \nabla \cdot\left(\rho D_{21}^{*} \nabla w\right)\right]\end{array}\right\} \times$

245

$\frac{1}{\frac{C_{v 0}}{C_{v i}}\left(\gamma_{0}-1\right)\left(\frac{\partial F}{\partial T}\right)_{\rho, w}\left(P_{t h}-T\left(\frac{\partial P_{t h}}{\partial T}\right)_{\rho, w}\right)-\rho^{2}\left(\frac{\partial F}{\partial \rho}\right)_{T, w}}$

with $A\left(\bar{U}^{*}, \bar{V}^{*}\right)=\left(\bar{U}_{2}^{*}-\bar{U}_{1}^{*}\right)+\frac{C_{v 0}}{C_{v i}}\left(\gamma_{0}-1\right)\left[P_{t h}-T\left(\frac{\partial P_{t h}}{\partial T}\right)_{\rho, w}\right] \times\left(\bar{V}_{2}^{*}-\bar{V}_{1}^{*}\right)$ 
247 The expressions of $\left(\frac{\partial F}{\partial T}\right)_{\rho, w},\left(\frac{\partial F}{\partial w}\right)_{T, \rho}$ and $\left(\frac{\partial F}{\partial \rho}\right)_{T, w}$ are reported in Appendix C. steps, namely the energy equation and the equation of state on the one hand and the NavierStokes equations on the other hand. The algorithm is detailed in section 2.4. observed.

$T_{i}=1+\mathcal{E}$

$\rho_{i}=1$

256

$w_{i}$ fixed

$$
P_{i}=\frac{(1+\varepsilon) \theta\left(w_{i}\right)}{1-b^{*}\left(w_{i}\right) / \theta\left(w_{i}\right)}-\frac{a^{*}\left(1+\varepsilon, w_{i}\right)}{1+2 b^{*}\left(w_{i}\right) / \theta\left(w_{i}\right)-b^{*}\left(w_{i}\right)^{2} / \theta\left(w_{i}\right)^{2}}
$$

No-slip walls were considered so $u=v=0$ at the two plates.

$$
\text { At } x=0 \quad T_{h}=1+\varepsilon+\delta T^{*}
$$




\subsection{Modeling of the adsorption reaction}

At the interfaces $\mathrm{x}=0$ and $H$, an adsorption reaction of Naphthalene on activated carbon is considered. The choice of such adsorption system can be explained by the extensive use of activated carbon as new-type high-efficiency adsorbent due to its high adsorption capacities and high mass transfer rates. However, the model description can be applied to any other adsorption system. The main objective here is to see how a supercritical mixture behaves in the vicinity of reactive wall. So, we will focus essentially on the fluid side rather than on what happens in the solid itself.

The species diffusion equation, (Eq. (4)), can be written in this form:

$\rho \frac{\partial w}{\partial t}+\rho u \frac{\partial w}{\partial x}+\rho v \frac{\partial w}{\partial y}=\nabla \cdot\left(\rho D_{21} \nabla w\right)$

where $u$ and $v$ are the velocity of components in the $x$-and $y$-directions respectively.

The boundary conditions are:

At the horizontal non-reactive walls

$$
\frac{\partial w}{\partial y}=0 \text { for } \mathrm{y}=0 \text { and } \mathrm{y}=H
$$

At the vertical reactive walls, a first order kinetic adsorption model is used:

$$
D_{21} \frac{\partial w}{\partial n}=K_{a} \cdot w
$$

where $\mathrm{n}$ is the normal to the surface at $\mathrm{x}=0$ and $\mathrm{x}=H$ and $\mathrm{K}_{\mathrm{a}}=\mathrm{k}_{\mathrm{a}} / \mathrm{S}_{\mathrm{ac}}$ with $\mathrm{k}_{\mathrm{a}}$ the adsorption rate constant $\left(\mathrm{m}^{3} \cdot \mathrm{kg}^{-1} \cdot \mathrm{s}^{-1}\right)$ and $\mathrm{S}_{\mathrm{ac}}$ the specific surface area of activated carbon $\left(\mathrm{m}^{2} \cdot \mathrm{kg}^{-1}\right)$.

Assuming small variations of temperature and pressure, $\mathrm{K}_{\mathrm{a}}$ can be approximated by the firstorder term of its Taylor series in the vicinity of $\left(\mathrm{T}_{\mathrm{i}}, \mathrm{P}_{\mathrm{i}}\right)$ :

$$
K_{a}=K_{a}\left(T_{i}, P_{i}\right)+\left(\frac{\partial K_{a}}{\partial T}\right)_{p}\left(T-T_{i}\right)+\left(\frac{\partial K_{a}}{\partial P}\right)_{T}\left(P-P_{i}\right)
$$


The adsorption rate constant $k_{a}$ can be computed by $k_{a}=K_{2} \cdot k_{d}$ where $K_{2}$ is the adsorption equilibrium constant $\left(\mathrm{m}^{3} \cdot \mathrm{kg}^{-1}\right)$ and $k_{d}$ is the desorption rate constant obtained through the Arrhenius law:

288

$k_{d}=A \exp \left(-\frac{E a}{R T}\right)$

with $E_{a}$ the activation energy and $A$ the pre-exponential factor of Arrhenius.

The first term of Eq. (9) can be then considered as:

291

$K_{a i}=K_{a}\left(T_{i}, P_{i}\right)=\frac{k_{d}\left(T_{i}\right) K_{2}\left(T_{i}, P_{i}\right)}{S_{a c}}$

292

The partial derivatives of $\mathrm{K}_{\mathrm{a}}$ with respect to temperature and pressure are written as follow:

$293\left(\frac{\partial K_{a}}{\partial T}\right)_{p}=\frac{1}{S_{a c}}\left(\frac{\partial k_{a}}{\partial T}\right)_{p}=\frac{k_{d}\left(T_{i}\right) K_{2}\left(T_{i}, P_{i}\right)}{S_{a c}}\left[\frac{E_{a}}{R T^{2}}+\left(\frac{\partial \operatorname{Ln} K_{2}}{\partial T}\right)_{p}\right]$

$294\left(\frac{\partial K_{a}}{\partial P}\right)_{T}=\frac{1}{S_{a c}}\left(\frac{\partial k_{a}}{\partial P}\right)_{T}=\frac{k_{d}\left(T_{i}\right) K_{2}\left(T_{i}, P_{i}\right)}{S_{a c}}\left(\frac{\partial \operatorname{Ln} K_{2}}{\partial P}\right)_{T}$

295 Thus Eq. (9) becomes:

296

$$
K_{a}(T, P)=K_{a i}\left\{1+\left[\frac{E_{a}}{R T^{2}}+\left(\frac{\partial \operatorname{Ln} K_{2}}{\partial T}\right)_{P}\left(T_{i}, P_{i}\right)\right]\left(T-T_{i}\right)+\left(\frac{\partial \operatorname{Ln} K_{2}}{\partial P}\right)_{T}\left(T_{i}, P_{i}\right)\left(P-P_{i}\right)\right\}
$$

297 The partial derivatives of $\mathrm{K}_{2}$ with respect to temperature and pressure are obtained by 298 equating differentials of the logarithm of the solute fugacity in the fluid and solid phases 299 [31]:

$300\left(\frac{\partial \ln K_{2}}{\partial T}\right)_{p}=\frac{\left(h_{2}^{I G}-\bar{h}_{2}^{m}\right)+\Delta H_{2}^{a d s}}{R T^{2}}+\alpha^{m}$ 
$\left(\frac{\partial \ln K_{2}}{\partial P}\right)_{T}=\frac{\bar{\vartheta}_{2}^{\infty, m}}{R T}-\kappa^{m}$

302

303

304

where $\Delta H_{2}^{a d s}=\bar{h}_{2}^{s}-h_{2}^{I G}$ is the heat of adsorption of the solute on the solid plate, $\bar{h}_{2}^{m}$ and $\bar{h}_{2}^{s}$ are the infinite-dilution partial molar enthalpies of solute in the mobile and stationary phases, respectively, $h_{2}^{I G}$ is the enthalpy of the solute in the ideal gas state, $\bar{\vartheta}_{2}^{\infty, m}$ is the infinitedilution partial molar volume of the solute in mobile phase and $\alpha^{m}, \kappa^{m}$ are respectively, the volume expansivity and the isothermal compressibility.

The infinite-dilution residual partial molar enthalpy $\left(h_{2}^{I G}-\bar{h}_{2}^{m}\right)$ of the solute, $\alpha^{m}, \kappa^{m}$ and $\bar{\vartheta}_{2}^{\infty, m}$ are obtained using the Peng-Robinson equation of state and they are reported in Appendix D.

As shown by Eq. (10), the derivatives of the equilibrium adsorption constant, $K_{2}$, with respect to temperature and pressure are directly involved in the definition of the boundary condition at the solid-fluid interface. So, it is important to assess the effect of temperature and pressure on these derivatives. A strong sensitivity to temperature and pressure can be guessed from Eqs. (11) - (12) since the infinite-dilution residual partial molar enthalpy, $\left(h_{2}^{I G}-\bar{h}_{2}^{m}\right)$, the infinite dilution partial molar volume of the solute, $\bar{\vartheta}_{2}^{\infty, m}$, the isothermal compressibility, $\kappa^{m}$, and the volume expansivity, $\alpha^{m}$, diverge near the solvent critical point. This assumption is confirmed by Fig. 2 which shows the $\mathrm{K}_{2}$ derivative profiles as a function of pressure for different temperatures. The profiles show a sharp minimum which becomes more important when the critical temperature is approached. Then this minimum is shifted to the high pressure domain and becomes less significant away from the critical point especially for $\mathrm{T}=318.15 \mathrm{~K}$. In this region (high pressure domain), the effect of the temperature on the isothermal derivative is less pronounced. In the same way, the isobaric derivative of the 
323

324

325

326

327

328

equilibrium constant as a function of temperature is shown in Fig. 3 for different pressures.

For pressures close to the critical one, namely $\mathrm{P} \leq 8.909 \mathrm{MPa}$, a divergence of the derivative is observed as the critical temperature is approached. Then, from $9 \mathrm{MPa}$, a behavior change can be depicted with the appearance of a maximum which decreases and is shifted to the high temperature domain when the pressure increases. One can also notice a similar trend for high pressures and high temperatures. Therefore beyond the critical point the effect of the pressure and temperature is no longer noticed.

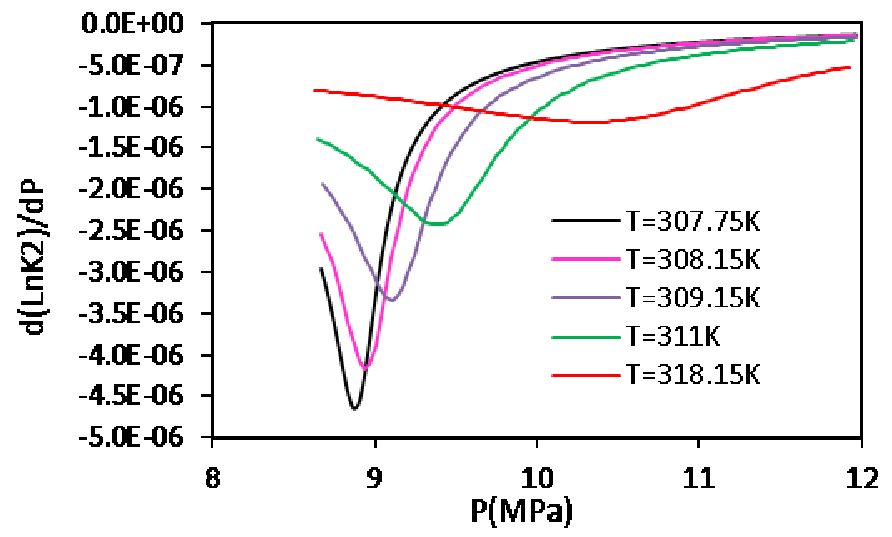

Fig. 2. Equilibrium constant derivative vs. pressure

330

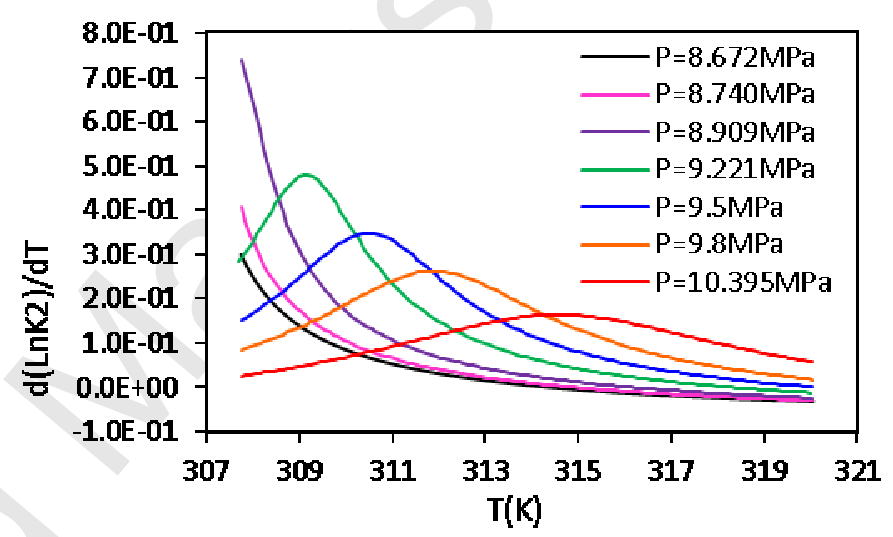

Fig 3. Equilibrium constant derivative vs. temperature

For this reason, a particular attention was paid to the effects of temperature and pressure on the equilibrium adsorption constant in this study because a wide range of temperature and pressure were considered. Moreover, changes near and far from the critical point will help us to explain our results later.

It must be noted that the divergence of the infinite-dilution properties (residual partial molar enthalpy and partial molar volume) of the solute is not specific of Naphthalene but it is a universal behavior for dilute mixtures [32-33]. Therefore, the results obtained in this paper for Naphthalene in supercritical $\mathrm{CO}_{2}$ are likely to be observed in all dilute binary mixtures, at least on a qualitative point of view. Moreover, the nature of the adsorbent material appears 
341

342

343

344

$D_{21} \frac{\partial w}{\partial n}=K_{a i} D_{k}\left(T, P_{t h}\right) w$ adsorbent material.

only through the heat of adsorption, $\Delta H_{2}^{a d s}$, in Eq. (11), and therefore just for the variation of $\mathrm{K}_{2}$ with respect to temperature. We compared the evolutions of $\mathrm{d}(\mathrm{LnK} 2) / \mathrm{dT}$ for soil $\left(\Delta H_{2}^{a d s}=\right.$ $-46054.8 \mathrm{~J} / \mathrm{mol})$ and for activated carbon $\left(\Delta H_{2}^{a d s}=-83736 \mathrm{~J} / \mathrm{mol}\right)$ as adsorbent material. The same variations were observed in both cases with maxima occurring for the same temperatures but with slightly different values: for example, the maximum for $\mathrm{P}=9.221 \mathrm{MPa}$ was equal to $0.52383 \mathrm{~K}^{-1}$ for soil and $0.47643 \mathrm{~K}^{-1}$ for activated carbon. These identical variations can be explained by the fact that the evolution of $d(\operatorname{LnK} 2) / d T$ is governed by the very large values of the infinite-dilution residual partial molar enthalpy, $\left(h_{2}^{I G}-\bar{h}_{2}^{m}\right)$, and of the volume expansivity, $\alpha^{m}$, near the critical point. And these two properties are completely independent of the characteristics of the adsorbent material. Consequently, the results presented in this paper for adsorption on activated carbon are also relevant for any other

In the framework of the Low Mach number approximation, the boundary condition for the mass fraction w on $\mathrm{x}=0$ and $H$ can then be expressed as:

with $D_{k}\left(T, P_{t h}\right)=1+\left[\frac{E_{a}}{R T^{2}}+\left(\frac{\partial \operatorname{Ln} K_{2}}{\partial T}\right)_{P}\left(T_{i}, P_{i}\right)\right]\left(T-T_{i}\right)+\left(\frac{\partial \operatorname{Ln} K_{2}}{\partial P}\right)_{T}\left(T_{i}, P_{i}\right)\left(P-P_{i}\right)$

357 In the dimensionless form, it will be written as:

$358-\frac{D_{21}^{*}}{D a} \frac{1}{D_{k}} \frac{\partial w^{*}}{\partial n}=1-w^{*}$ 
with $D_{21}^{*}=D_{21} /\left(D_{21}\right)_{i}, w^{*}=\left(w-w_{i}\right) / w_{i}$ and Da is the Damköhler number defined as the ratio of the characteristic fluidic time scale (diffusion characteristic time) and chemical time scale (adsorption characteristic time):

$D a=\frac{H K_{a i}}{\left(D_{21}\right)_{i}}$

Eq. (13) leads to two Robin-type boundary conditions at $\mathrm{x}=0$ and $\mathrm{x}=1$ :

At $x=0 w^{*}+\frac{D_{21}^{*}}{D a} \frac{1}{D_{k}} \frac{\partial w^{*}}{\partial x}=1$

At $x=1$, the wall is maintained at the initial temperature, thus, $D_{21}^{*}=1$ :

$w^{*}-\frac{1}{D a} \frac{1}{D_{k}} \frac{\partial w^{*}}{\partial x}=1$

\subsection{Numerical method}

The numerical integration of the model equations has been carried out using a second order semi-implicit scheme [34]: the convective terms are evaluated by an Adams-Bashforth scheme, and then the time integration of the resulting differential equations has been done with an implicit second order backward Euler scheme. The space approximation is performed using the Chebyshev-collocation method with Gauss-Lobatto points. For the computation of the convective terms, the derivatives are calculated in the spectral space and the products are performed in the physical one; the connection between the spectral and the physical spaces is realized through a FFT algorithm. On the other hand, the spectral differentiation matrices are used for the derivatives in the diffusive terms.

The computation of the velocity divergence by Eq. (8) allowed a decoupling between the thermodynamic variables $T, \rho, P_{t h}, w^{*}$ and that of the dynamic field. Consequently, the discretized equations can be solved in two successive steps: first, the thermodynamic variables are computed through the algorithm proposed by Ouazzani and Garrabos [29] and 
then the Navier-Stokes equations are solved using the modified projection method developed in [35] and extended to variable density flows. These two steps are detailed bellow.

\subsubsection{Computation of the thermodynamic variables $\left(T, \rho, P_{t h}, w^{*}\right)$}

The discretised energy and diffusion equations can be written as Helmholtz equations with time-dependent coefficients. In order to solve them using the diagonalization technique developed in [36] for Helmholtz equations with constant coefficients, the density and the transport coefficients $\lambda^{*}$ and $D_{21}^{*}$ are split into a constant part equal to the initial value and a time-dependent part:

$\alpha^{n+1}=1+\left(\alpha^{n+1}-1\right)$ for $\alpha=\rho, \lambda^{*}, D_{21}^{*}$

So, the discretized energy equation for example obtained as Helmholtz equation with constant coefficients is written as follow:

$$
\begin{aligned}
\frac{\gamma}{\operatorname{Re} \operatorname{Pr}} \Delta T^{n+1}-\frac{3}{2 \delta t} T^{n+1} & =\frac{3}{2 \delta t}\left(\rho^{n+1}-1\right) T^{n+1}-\frac{\gamma}{\operatorname{Re} \operatorname{Pr}} \nabla \cdot\left(\left(\lambda^{* n+1}-1\right) \nabla T^{n+1}\right) \\
& +\rho^{n+1}\left(\frac{-4 T^{n}+T^{n-1}}{2 \delta t}\right)+A B(\rho \mathbf{V} \cdot \nabla T)^{n, n-1} \\
& +\frac{C_{v 0}}{C_{v i}}\left(\gamma_{0}-1\right)\left[P_{t h}^{n+1}-T^{n+1}\left(\frac{\partial P_{t h}}{\partial T}\right)_{\rho, w}^{n+1}\right](\nabla \cdot V)^{n+1} \\
& -\frac{w_{i}}{\theta\left(w^{n+1}\right)} A\left(\bar{U}^{* n+1}, \bar{V}^{* n+1}\right) \frac{1}{(\gamma-1)^{2} L e} \nabla \cdot\left(\rho^{n+1} D_{21}^{* n+1} \nabla^{{ }^{*} n+1}\right)
\end{aligned}
$$

where $\delta t$ is the time-step and the notation $A B($.$) means an Adams-Bashforth evaluation of the$ quantity:

$$
A B(\phi)^{n, n-1}=2 \phi^{n}-\phi^{n-1} .
$$

The diagonalization process of the Helmholtz operator with constant coefficients is executed only once in a preprocessing stage. After that, at each time step, the solution of Eq. (16) is reduced to matrix products, leading to a very efficient solution technique. 
Thus, the solution of the energy Eq. (16), the diffusion equation and the equation of state is performed through the following iterative algorithm:

1. The variables $T^{k-1}, P_{t h}^{k-1}, \rho^{k-1}, w^{*(k-1)}$ and $(\nabla \cdot V)_{T}^{k-1}$ are initialized at their values at the previous time step $n$;

2. The temperature $T^{k}$ is obtained by the solution of the Helmholtz equation:

405

406

$$
\begin{aligned}
\frac{\gamma}{\operatorname{Re} \operatorname{Pr}} \Delta T^{k}-\frac{3}{2 \delta t} T^{k} & =\frac{3}{2 \delta t}\left(\rho^{k-1}-1\right) T^{k-1}-\frac{\gamma}{\operatorname{Re} \operatorname{Pr}} \nabla \cdot\left(\left(\lambda^{k-1}-1\right) \nabla T^{k-1}\right) \\
& +\rho^{k-1}\left(\frac{-4 T^{n}+T^{n-1}}{2 \delta t}\right)+A B(\rho \mathbf{V} \cdot \nabla T)^{n, n-1}
\end{aligned}
$$

$$
\begin{aligned}
& +\frac{C_{v 0}}{C_{v i}}\left(\gamma_{0}-1\right)\left[P_{t h}^{k-1}-T^{k-1}\left(\frac{\partial P_{t h}}{\partial T}\right)_{\rho, w}^{k-1}\right](\nabla \cdot V)^{k-1} \\
& -\frac{w_{i}}{\theta\left(w^{k-1}\right)} A\left(\bar{U}^{* k-1}, \bar{V}^{* k-1}\right) \frac{1}{(\gamma-1)^{2} L e} \nabla \cdot\left(\rho^{k-1} D_{21}^{* k-1} \nabla w^{* k-1}\right)
\end{aligned}
$$

$$
w^{* *}-\frac{1}{D a} \frac{\partial w^{* k}}{\partial x}=1-\frac{1}{D a}\left(1-\frac{1}{D_{k}^{k-1}}\right) \frac{\partial w^{*(k-1)}}{\partial x} \quad \text { for } \mathrm{x}=1
$$

with the following Robin boundary conditions on adsorbing walls:

$$
w^{* k}+\frac{1}{D a} \frac{\partial w^{* k}}{\partial x}=1+\left(\frac{1}{D a}-\frac{D_{21}^{* k-1}}{D a} \frac{1}{D_{k}^{k-1}}\right) \frac{\partial w^{*^{(k-1)}}}{\partial x} \text { for } \mathrm{x}=0
$$

3. The mass fraction $w^{* k}$ is obtained by the solution of the Helmholtz equation:

$$
\begin{aligned}
& \frac{1}{(\gamma-1)^{2} L e} \Delta w^{{ }^{* k}}-\frac{3}{2 \delta t} w^{{ }^{* k}}=\frac{3}{2 \delta t}\left(\rho^{k-1}-1\right) w^{{ }^{* k-1}}-\frac{1}{(\gamma-1)^{2} L e} \nabla \cdot\left(\left(\rho^{k-1} D_{21}^{*_{k}-1}-1\right) \nabla w^{{ }^{* k-1}}\right) \\
& +\rho^{k-1}\left(\frac{-4 w^{{ }^{*} n}+w^{*_{n-1}}}{2 \delta t}\right)+A B\left(\rho V \nabla w^{*}\right)^{n, n-1}
\end{aligned}
$$

4. The couple $\left(P_{t h}^{k}, \rho^{k}\right)$ is computed from the constraint of global mass conservation and the equation of state. This computation must be performed through an iterative process; 
5. The thermal conductivity $\lambda^{* k}$ and the diffusion coefficient $D_{21}^{* k}$ are updated.

6. The velocity divergence $(\nabla \cdot V)_{T}^{k}$ is computed by Eq. (8).

The steps 2 to 6 are repeated until convergence is achieved on temperature, thermodynamic pressure, density and mass fraction. The convergence criterion used is $\operatorname{Max}(\operatorname{Res} T$, Res ,Resw, $\left.\operatorname{Res} P_{t h}\right)<10^{-11}$, with $\operatorname{Res}=\operatorname{Max}\left(\left({ }^{k}{ }^{k-1}\right) /^{k-1}\right)$ for $=T,, w^{*}, P_{t h}$, and the maximum number of iterations is fixed to 250 .

\subsubsection{Solution of the Navier-Stokes equations}

The second step is the solution of the Navier-Stokes equations. At the current time step $(n+1)$, temperature, density and velocity divergence are known. A projection-type algorithm such as those developed for the solution of incompressible Navier-Stokes equations can then be used. In the present work, the original projection method of Hugues and Randriamampianina [35] was modified to account for variable density flows [30, 39]. The advantage of this method compared to other projection methods is that it allows improving the accuracy on pressure and reducing the slip velocity. It consists in solving the Navier-Stokes equations by three successive steps.

\section{$\underline{I^{s t} \text { Step: } \text { Computation of a preliminary pressure }}$}

The preliminary pressure $\bar{P}_{d y n}^{n+1}$ is computed from a Poisson equation, derived from the discretized momentum equation, with Neumann boundary conditions obtained by the normal projection of the momentum equation on the boundary: 


$$
\left\{\begin{aligned}
\Delta \bar{P}_{d y n}^{n+1}= & \nabla \cdot\left[-A B(\rho \mathbf{V} \cdot \nabla \mathbf{V})^{n, n-1}+\rho^{n+1}\left(\frac{4 \mathbf{V}^{n}-\mathbf{V}^{n-1}}{2 \delta t}\right)+\frac{1}{F r} \rho^{n+1} g\right] \\
& +\frac{4}{3 \operatorname{Re}} \Delta(\nabla \cdot \mathbf{V})_{T}^{n+1}+\frac{3}{2 \delta t}\left(\frac{3 \rho^{n+1}-4 \rho^{n}+\rho^{n-1}}{2 \delta t}\right) \\
\frac{\partial \bar{P}_{d y n}^{n+1}}{\partial n}= & n \cdot\left[-A B(\rho \mathbf{V} \cdot \nabla \mathbf{V})^{n, n-1}-\rho^{n+1}\left(\frac{3 \mathbf{V}_{B}^{n+1}-4 \mathbf{V}^{n}+\mathbf{V}^{n-1}}{2 \delta t}\right)+\frac{1}{F r} \rho^{n+1} g\right. \\
& \left.+\frac{4}{3 \operatorname{Re}} \nabla(\nabla \cdot \mathbf{V})_{T}^{n+1}-\frac{1}{\operatorname{Re}} A B(\nabla \times(\nabla \times \mathbf{V}))^{n, n-1}\right]
\end{aligned}\right.
$$

on $\partial \Omega$

439

with $\Omega$ the computational domain $(\Omega=]-1,+1[\times]-1,+1[), \partial \Omega$ its boundary, $V_{B}^{n+1}$ the boundary

conditions of the velocity $\boldsymbol{V}^{n+1}, \partial / \partial n$ the normal derivative and $\nabla . \mathbf{V}$ is calculated from thermodynamic variables and noted $(\nabla \cdot \mathbf{V})_{T}$.

$$
\Delta \boldsymbol{V}^{n+1}=\nabla\left(\nabla \cdot \boldsymbol{V}^{n+1}\right)-\nabla \times\left(\nabla \times \boldsymbol{V}^{n+1}\right)
$$

and the rotational term was evaluated using an Adams-Bashforth scheme.

\section{$\underline{2^{\text {nd }} \text { Step: Computation of a predicted velocity } \boldsymbol{V}^{*}}$}

The predicted velocity field $\boldsymbol{V}^{*}$ is computed implicitly from the momentum equation with

$$
\begin{cases}\rho^{n+1} \frac{3 \mathbf{V}^{*}-4 \mathbf{V}^{n}+\mathbf{V}^{n-1}}{2 \delta t}+A B(\rho \mathbf{V} . \nabla \mathbf{V})^{n, n-1}=-\nabla \bar{P}_{d y n}^{n+1}+\frac{1}{\operatorname{Re}} \Delta \mathbf{V}^{*}+\frac{1}{3 \operatorname{Re}} \nabla(\nabla . V)_{T}^{n+1}+\frac{1}{F r} \rho^{n+1} g & \text { in } \Omega \\ \mathbf{V}^{*}=\mathbf{V}_{B}^{n+1} & \text { on } \partial \Omega\end{cases}
$$


Here again, we have to solve Helmholtz equations with variable coefficients for each

459 velocity component. As for energy and mass diffusion equations, the density $\rho^{n+1}$ is split into coefficient is solved iteratively:

462

$$
\begin{aligned}
\frac{1}{\operatorname{Re}} \Delta \mathbf{V}^{*, l}-\frac{3}{2 \delta t} \rho_{0} \mathbf{V}^{*, l} & =\frac{3}{2 \delta t}\left(\rho^{n+1}-\rho_{0}\right) \mathbf{V}^{*, l-1}+\nabla \bar{P}_{d y n}^{n+1} \\
& -\rho^{n+1}\left(\frac{4 \mathbf{V}^{n}-\mathbf{V}^{n-1}}{2 \delta t}\right)+A B(\rho \mathbf{V} \cdot \nabla \mathbf{V})^{n, n-1} \\
& -\frac{1}{3 \operatorname{Re}} \nabla(\nabla \cdot V)_{T}^{n+1}-\frac{1}{F r} \rho^{n+1} g
\end{aligned}
$$

463

The convergence is achieved when $\operatorname{Max}(\operatorname{Resu}, \operatorname{Resv})<10^{-13}$, with $\operatorname{Res}=\operatorname{Max}\left(\left({ }^{l}-{ }^{l-1}\right) /^{l-1}\right)$ for $=u, v$. Only 3 or 4 iterations are necessary.

466

467

$\underline{3^{\text {nd }} \text { Step: } \text { Correction step }}$

468

The converged velocity field $\boldsymbol{V}^{*}$ is then corrected by taking into account the pressure 469 gradient at the current time step $(n+1)$ so that the final velocity field satisfies the continuity

470 equation:

471

472

$$
\left\{\begin{array}{l}
\frac{3}{2 \delta t}\left(\rho^{n+1} \boldsymbol{V}^{n+1}-\rho^{n+1} \boldsymbol{V}^{*}\right)=-\nabla\left(P_{d y n}^{n+1}-\bar{P}_{d y n}^{n+1}\right) \quad \text { in } \Omega \cup \partial \Omega \\
\boldsymbol{V}^{n+1} . n=\boldsymbol{V}_{B}^{n+1} . n \quad \text { on } \partial \Omega \\
\frac{3 \rho^{n+1}-4 \rho^{n}+\rho^{n-1}}{2 \delta t}+\nabla \cdot\left(\rho^{n+1} \boldsymbol{V}^{n+1}\right)=0 \quad \text { in } \Omega
\end{array}\right.
$$

473

474 This system is solved through the following Poisson problem for the intermediate $475 \quad$ variable $\varphi=2 \delta t\left(P_{d y n}^{n+1}-\bar{P}_{d y n}^{n+1}\right) / 3$ : 
$477 \begin{cases}\Delta \varphi=\nabla \cdot\left(\rho^{n+1} V^{*}\right)+\frac{3 \rho^{n+1}-4 \rho^{n}+\rho^{n-1}}{2 \delta t} & \text { in } \Omega \\ \frac{\partial \varphi}{\partial n}=0 & \text { on } \partial \Omega\end{cases}$

478

479 The actual velocity field and pressure at the current time step $(n+1)$ are finally calculated in $480 \Omega \cup \partial \Omega$ by the formulae:

$481 \quad V^{n+1}=V^{*}-\frac{1}{\rho^{n+1}} \nabla \varphi$

$482 \quad P_{d y n}^{n+1}=\bar{P}_{d y n}^{n+1}+\frac{3}{2 \delta t} \varphi$

483

484

485

486

487

488

489

490

491

492

493

494

495

496

497 


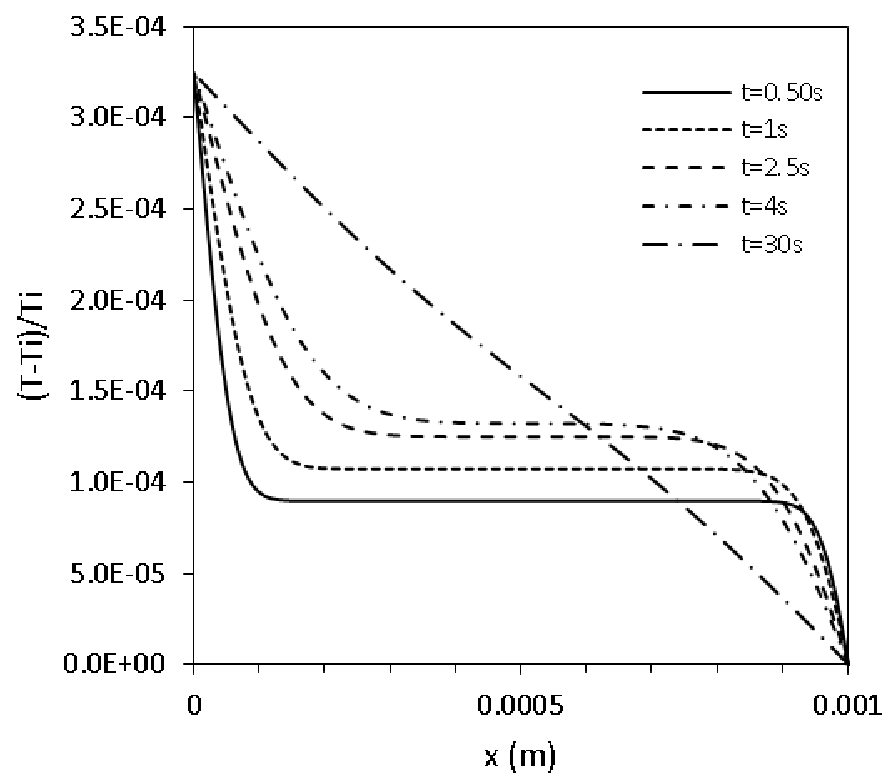

499

500

501

502

503

504

505

506

507

508

509

510

511

512

513

514

515

516

517

518

519

520

521

522

523

524

525

526

527

528

529

530

Fig. 4. Profiles of the temperature perturbation at several times for $\mathrm{T}_{\mathrm{i}}=308.15 \mathrm{~K}$ and $\Delta \mathrm{T}=100 \mathrm{mK}$ in the case $\mathrm{g}=0$

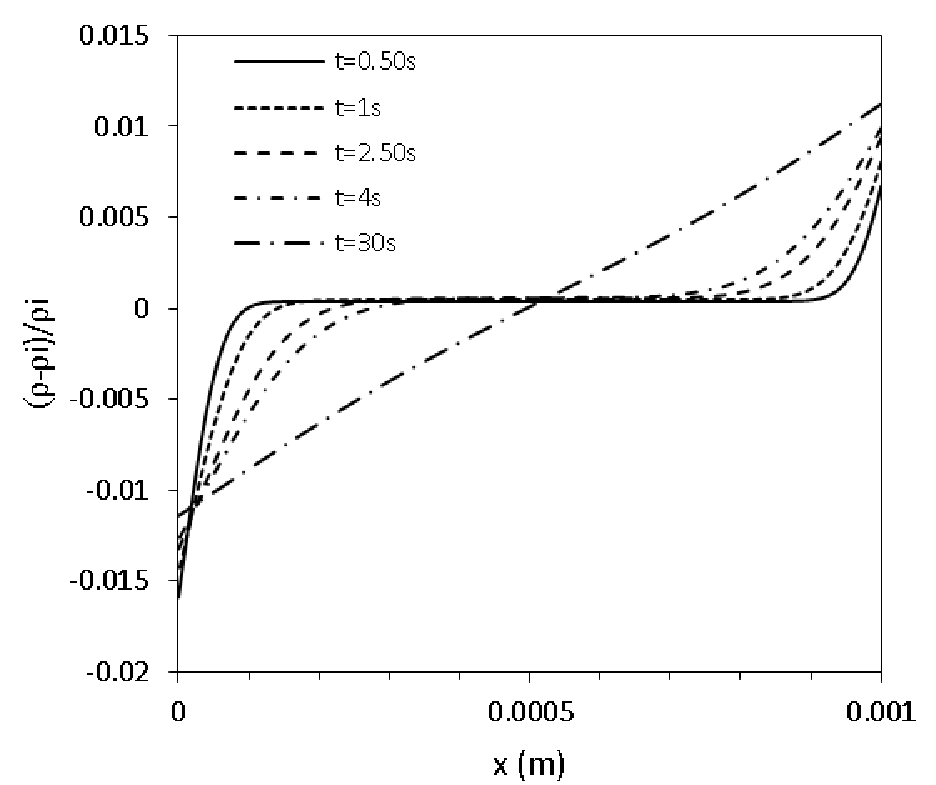

Fig. 5. Profiles of the density perturbation at several times for $\mathrm{Ti}=308.15 \mathrm{~K}$ and $\Delta \mathrm{T}=100 \mathrm{mK}$

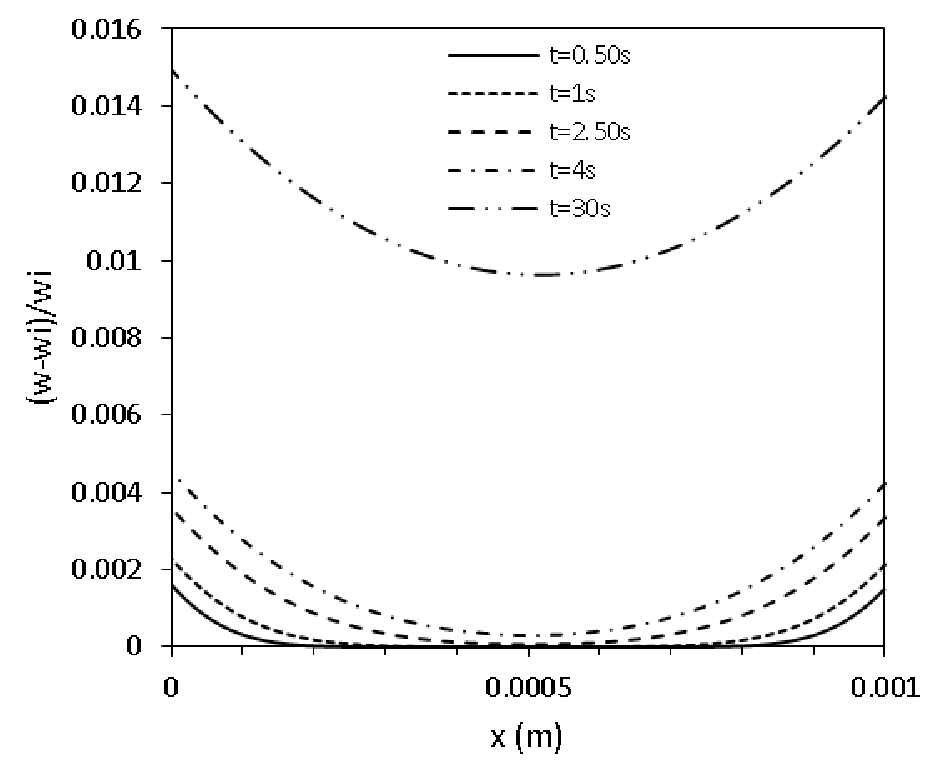

Fig. 6. Profiles of the mass fraction perturbation at several times for $\mathrm{T}_{\mathrm{i}}=308.15 \mathrm{~K}$ and $\Delta \mathrm{T}=100 \mathrm{mK}$ in the case $\mathrm{g}=0$

The system's response to the heating of the left vertical wall is explained in this section. The analysis below is based on simulations carried out at various initial temperatures 
$T_{i}$ ranging from $307.75 \mathrm{~K}$ to $318.15 \mathrm{~K}$ and which correspond to dimensionless distances $\varepsilon$ to the critical point ranging from $3.25 \times 10^{-4}$ to $3.41 \times 10^{-2}$. The values of the different characteristic times of the problem are given in Table 3. It can be noted that, as the critical temperature is approached, the thermal diffusion and mass diffusion times strongly increase whereas the characteristic time of piston effect decreases. For all the simulations, the left side of the cavity (at $\mathrm{x}=0$ ) is gradually heated following a cosine low expressed as:

$$
\delta T(t)= \begin{cases}0.5 \Delta T\left(1-\cos \left(\pi \frac{t}{t_{\text {heat }}}\right)\right) & \text { if } t \leq t_{\text {heat }} \\ \Delta T & \text { if } t \geq t_{\text {heat }}\end{cases}
$$

where $\Delta T$ is the temperature increase and $\mathrm{t}_{\text {heat }}$ is the heating phase time corresponding to 200 time steps. The influence of $\Delta T$ will be discussed later for different cases $(\Delta T=50,100,150$ and $200 \mathrm{mK})$.

\subsection{General description}

In the first part, the case of $T_{i}=308.15 \mathrm{~K}$ and $\Delta T=100 \mathrm{mK}$, is discussed. The Damköhler number is fixed to $\mathrm{Da}=10^{-5}$ and the initial mass fraction $\mathrm{w}_{\mathrm{i}}$ corresponds to the solubility of Naphthalene in $\mathrm{CO}_{2}$.

The evolution of temperature and density distributions between the walls and at the cavity mid-height are illustrated in Figs. 4 and 5 for several times $(t=0.5 \mathrm{~s}, 1 \mathrm{~s}, 2.5 \mathrm{~s}, 4 \mathrm{~s}$ and $30 \mathrm{~s})$ in the absence of gravity. Because of the very small thermal diffusivity near the critical point, the heating of the wall causes ultra-thin boundary layers at the wall-fluid interface. Due to the high isothermal compressibility, the fluid close to the heated side expands upward and converts some of the kinetic energy into thermal energy. This results in compressing adiabatically the rest of the fluid and leading to a quick increase of the thermodynamic pressure which induces a fast and homogeneous heating of the cavity bulk by thermo-acoustic 
effects (piston effect). During the heating phase, temperature, and therefore density, at the heated wall change at each time step and, as a consequence, temperature and density gradients near this wall increase more and more. As the bulk temperature grows, and since the right wall (at $\mathrm{x}=H$ ) temperature is maintained at its initial value, a cold boundary layer settles near the right wall where the fluid contracts. The contraction causes an expansion of the bulk and reduces the bulk temperature. The behavior of the fluid is the result of these two competing processes, heating by hot boundary layer and cooling by cold boundary layer. The temperature field is then divided into three distinct zones: two thermal boundary layers associated with large density gradients (Fig. 5) along the vertical walls and the isothermal cavity bulk. Therefore, the temperature and the density profiles exhibit the same behavior, dominated by the piston effect, as in pure fluid [45-46]. In the rest of the paper, we denote as bulk the fluid region, which does not include the boundary layers. The homogeneous bulk temperature and density fields of a supercritical fluid occur at a time that is much shorter than the thermal diffusion time $\left(t_{P E}=0.23 \mathrm{~s}\right.$ and $\left.t_{d}=107 \mathrm{~s}\right)$. They are the signature of the piston effect which was identified a long time ago as responsible for fast heat transport in near-critical pure fluids [47-49]. However, the piston effect plays an important role only for short times because of the disappearance of sharp temperature gradients due to the action of thermal diffusivity. For an advanced time (30s), the system's response, is then markedly different from that observed for shorter times. A similar trend to that of a perfect gas characterized by equilibrium can be depicted. It must be noted that temperature equilibrium is achieved on a time much shorter than the diffusion time. This is probably a consequence of the evolution of mass fraction in the cavity bulk, since w influences the temperature evolution as a source term of the energy equation.

The mass fraction field exhibits a different aspect as shown in Fig. 6. In order to explain the typical behavior of the supercritical mixture near the two reactive walls, we focus on the 
579 boundary conditions developed in section 2.3. The dependence to temperature and pressure of

580 the adsorption rate can be depicted from Eq. (10). Since the partial derivative of $\mathrm{K}_{2}$ with 581 respect to pressure is negative (see Fig. 2), the pressure term tends to reduce $K_{a}$ and as a 582 result, to diminish the adsorbed amount. On the left heated wall, the effect of the temperature 583 increase is amplified by the diverging derivative of $\mathrm{K}_{2}$ with respect to temperature (see Fig. 584 3), leading to an important amount of Naphthalene adsorbed at the warm side.

585 The phenomenon occurring at the isothermal right wall is totally different. The strong density 586 gradient near the boundary $\mathrm{x}=H$ (Fig. 5), generated by the piston effect, goes along with an 587 increase of the amount of Naphthalene neat this reactive wall leading to an increase of the 588 adsorbed quantity. Finally, the strong and homogeneous increase of the pressure, induced by 589 the piston effect in the whole cavity, reduces the adsorbed amount at both reactive walls. 


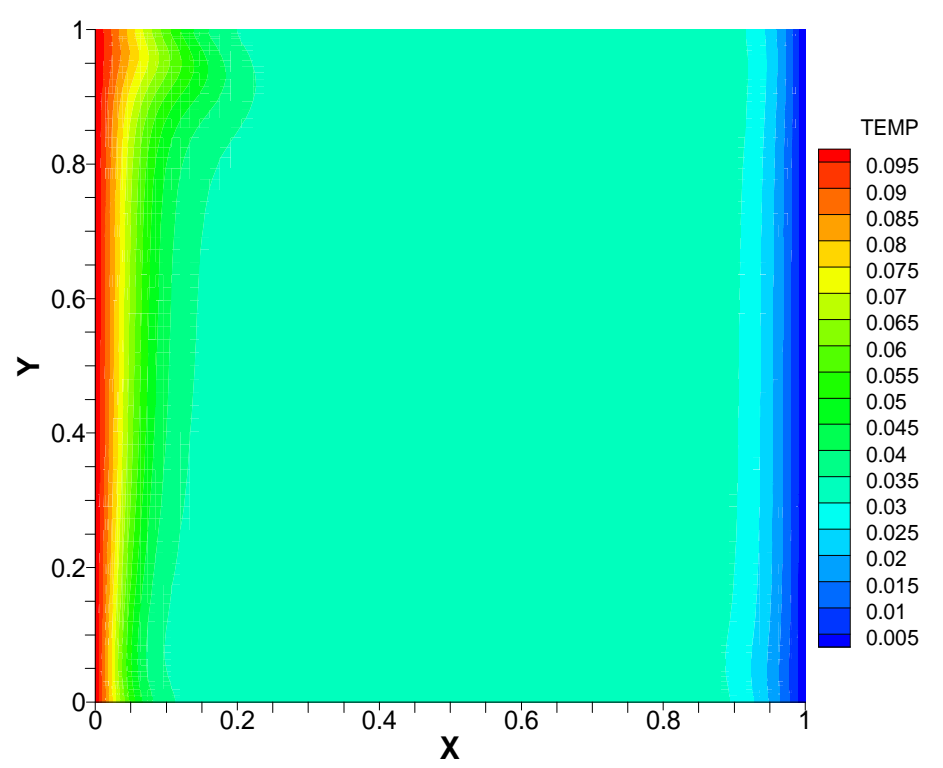

(a)

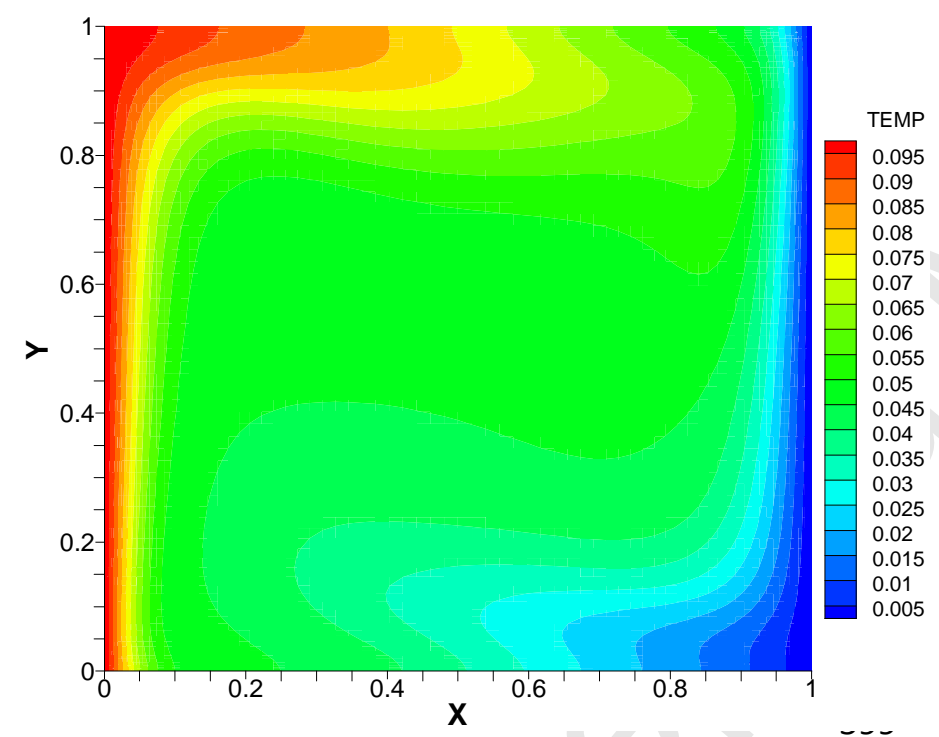

(c)

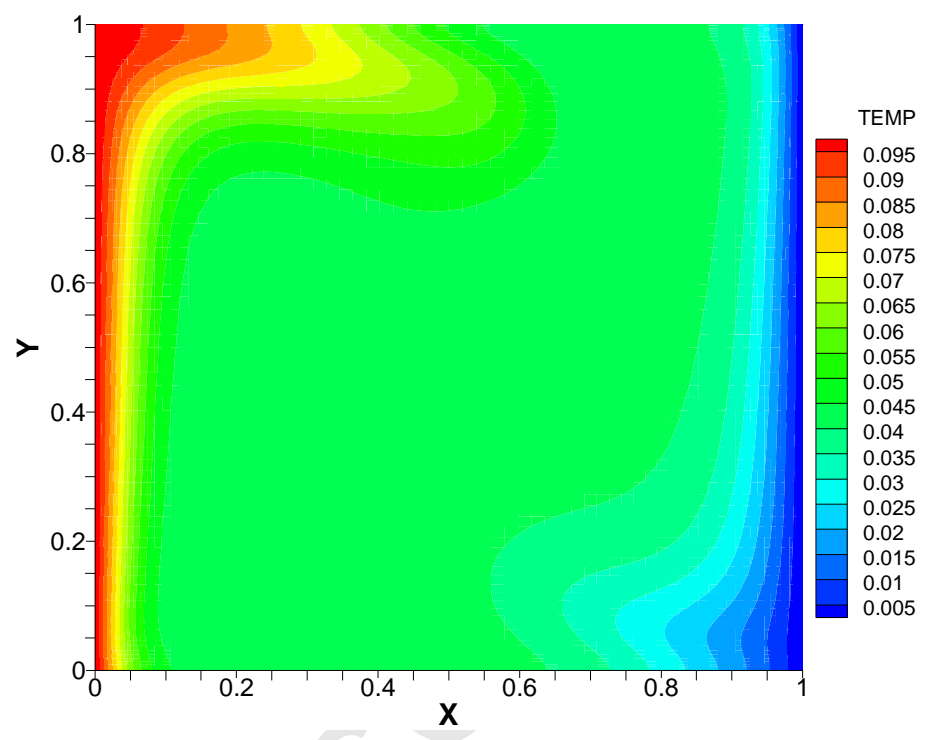

(b)

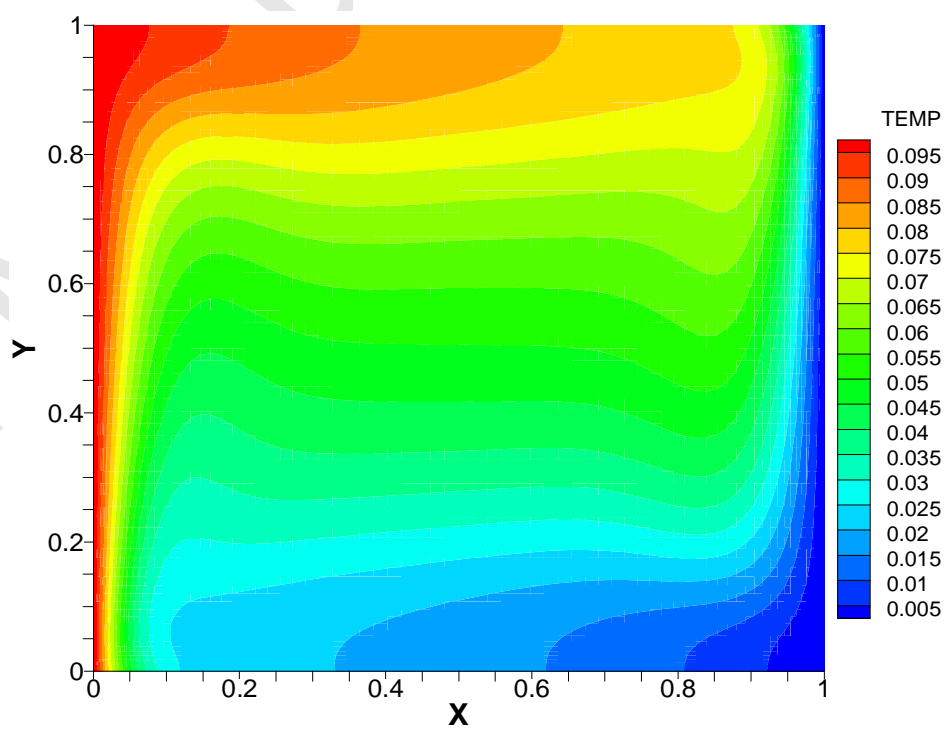

(d)
600

601

602

603

604

605 density fields obtained are completely two-dimensional on the contrary to the mainly 1D

606 solutions previously observed in the absence of gravity. In Fig. 7, instantaneous temperature

607 fields are plotted in the (x,y) plan for several times (1 s, $2.5 \mathrm{~s}, 4 \mathrm{~s}$ and $30 \mathrm{~s})$. During a short

Fig. 7. Isosurfaces of the temperature variation for several times (a) $1 \mathrm{~s}$, (b) $2.5 \mathrm{~s}$, (c) $4 \mathrm{~s}$ and (d) $30 \mathrm{~s}$, for $\mathrm{T}_{\mathrm{i}}=308.15 \mathrm{~K}$ and $\Delta \mathrm{T}=100 \mathrm{mK}$ in the presence of gravity

We consider now the case when the mixture is subjected to the gravity. In this case, side heating initiates gravity-driven convection in the fluid phase and the temperature and time, the cavity bulk is heated rapidly due to the piston effect. As a result, upstream rises near 
the left warm surface and a hot spot at the left corner of the cavity can be depicted after 1s and then it is convected progressively along the top wall for longer times. As in Fig. 4 for g=0, a cool boundary layer forms near the right isothermal wall, due to the piston effect. As a result, a jet moving down appears near the right wall from $2.5 \mathrm{~s}$. The hot and cold thermal plumes along the top and the bottom sides develop progressively in time as shown in Fig. 7. We can clearly see the homogenous increase of the bulk temperature induced by the piston effect. This aspect is different from the perfect gas case where thermal boundary layer is formed only near heated side which leads to a single stream [50].

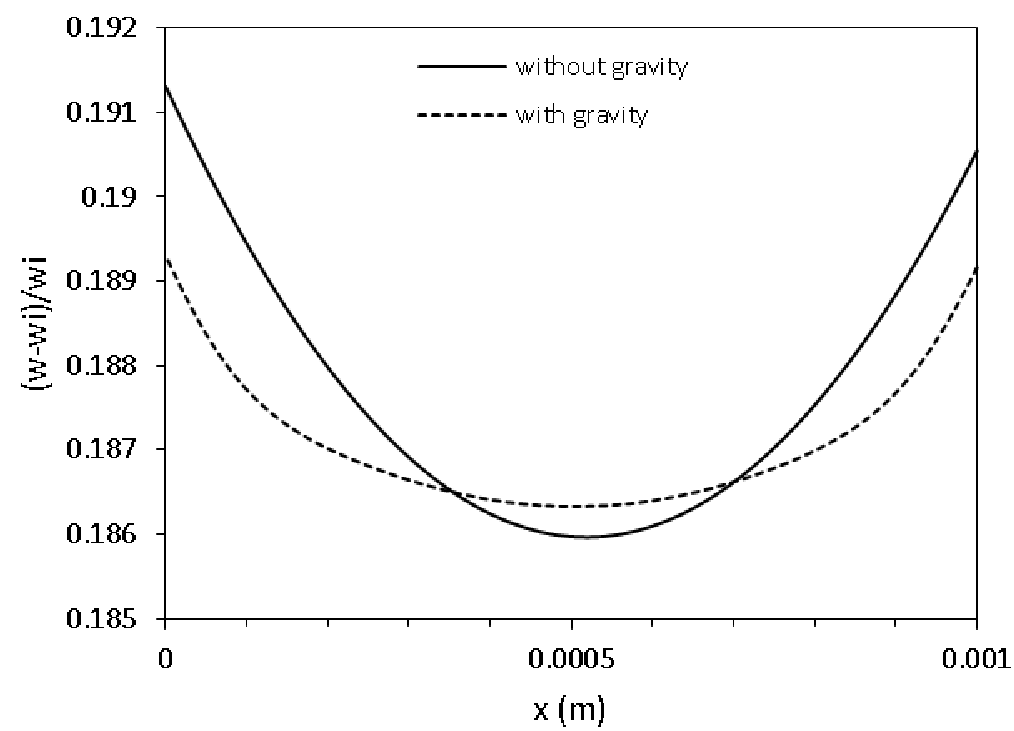

Fig. 8. The effect of the gravity on the mass fraction perturbation for $\mathrm{T}_{\mathrm{i}}=308.15 \mathrm{~K}$ and $\Delta \mathrm{T}=100 \mathrm{mK}$ at $\mathrm{t}=500 \mathrm{~s}$

For the sake of comparison and in order to highlight the effect of Earth's gravity, we analyze in the following the Naphthalene mass fraction evolution with and without gravity (Fig. 8). The difference previously observed between the hot wall and the cold wall has disappeared. Gravity in this case tends to balance both sides. Conversely, the amount adsorbed is remarkably lower at the two sides (hot and cold) compared to the case without gravity. On the other hand, the mass fraction at the cavity centre has increased. These observations can be explained as follow: 
627 For the case without gravity, thermal diffusion process is the only highlighted. The

628

629

630

631

632

633

634

635

characteristic time of thermal diffusion is much higher than the time scale of the piston effect.

Such a long process allows keeping a high temperature in the vicinity of the heated wall.

Consequently, as seen above, the divergence of the partial derivative of the equilibrium

constant with respect to temperature near the critical point is believed to affect the mass

fraction rate at the hot side. However, in the case with gravity, density variations generate a plume which expands upwardly (as shown in Fig. 7). This intensive plume moves hot fluid to the top boundary and thus decreases the temperature near the warm side. Therefore, the effect of the derivative with respect to temperature is reduced. Such a phenomenon can explain the reduction of the mass fraction when gravity is taken into account.

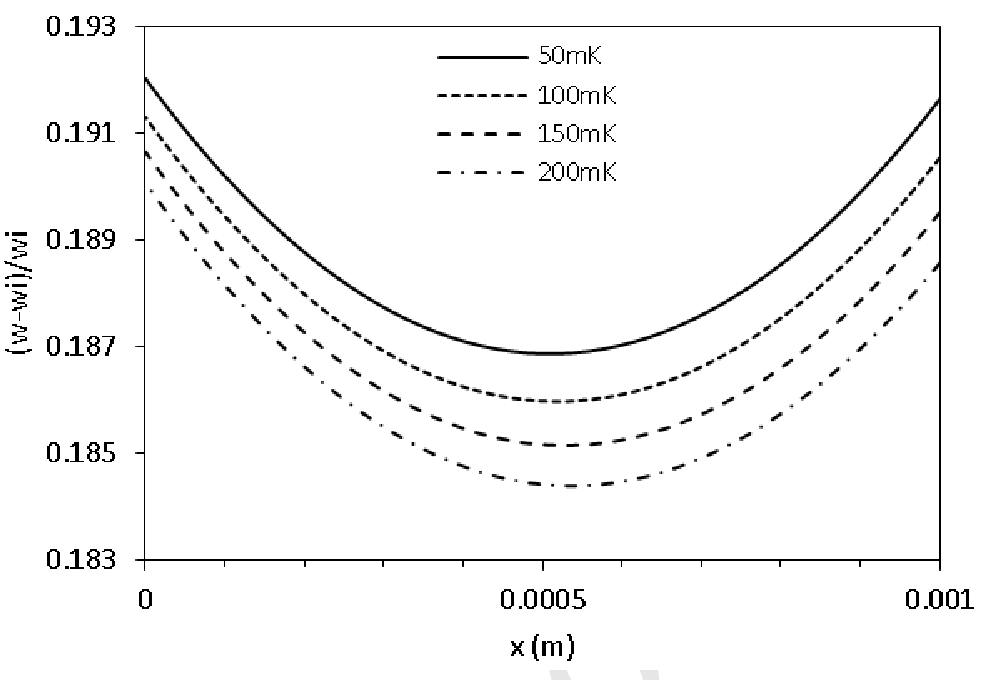

(a)

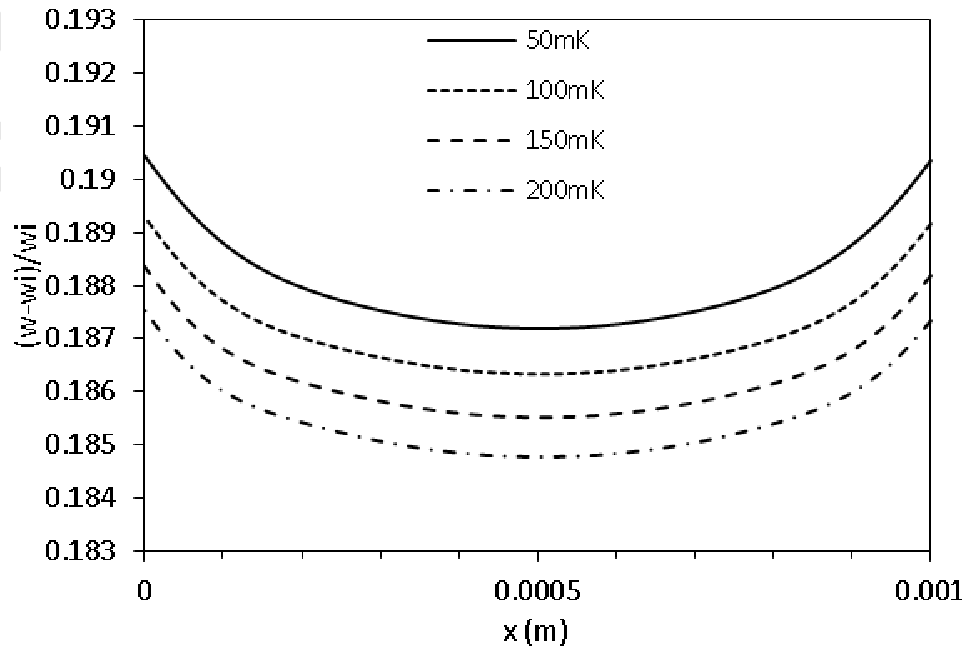

(b)
637

638

639

640

641

642

643

644

Fig. 9. The effect of the solid plate temperature on the mass fraction perturbation without gravity (a) and with gravity (b) for $\mathrm{T}_{\mathrm{i}}=308.15 \mathrm{~K}$ at $\mathrm{t}=500 \mathrm{~s}$

The effect of heating intensity is highlighted in Fig. 9 with and without gravity. For the same proximity to the critical point and the same time, Fig. 9 shows a comparison of the mass fraction profiles at the cavity mid-height obtained for four values of the temperature rise $\Delta \mathrm{T}=$ $50 \mathrm{mK}, 100 \mathrm{mK}, 150 \mathrm{mK}$ and $200 \mathrm{mK}$ : the mass fraction decreases with increasing heating. In the case without gravity, a stronger heating reduces remarkably the mass fraction throughout the entire volume while keeping the asymmetry of adsorbed amount between the hot and the 
cold walls (Fig. 9(a)). It can be noted that this asymmetry increases with the heating. A

646 similar trend can be observed with gravity (Fig. 9(b)). This surprising result can be explained

647 by the negative pressure term in the adsorption rate $\mathrm{K}_{\mathrm{a}}$ (Eq. (10)). Indeed, a stronger heating

648 of the wall induces a larger thermodynamic pressure increase by the piston effect and, as a

649 result, a larger value of the pressure term which reduces the parameter $\mathrm{K}_{\mathrm{a}}$. The reduction of

650 the adsorbed amount at the walls then leads to a smaller mass fraction in the whole cavity.

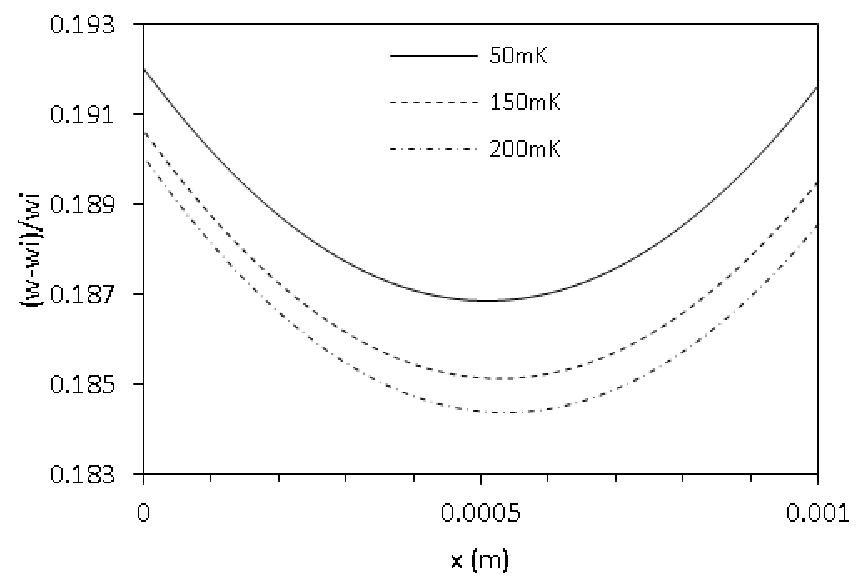

(a)

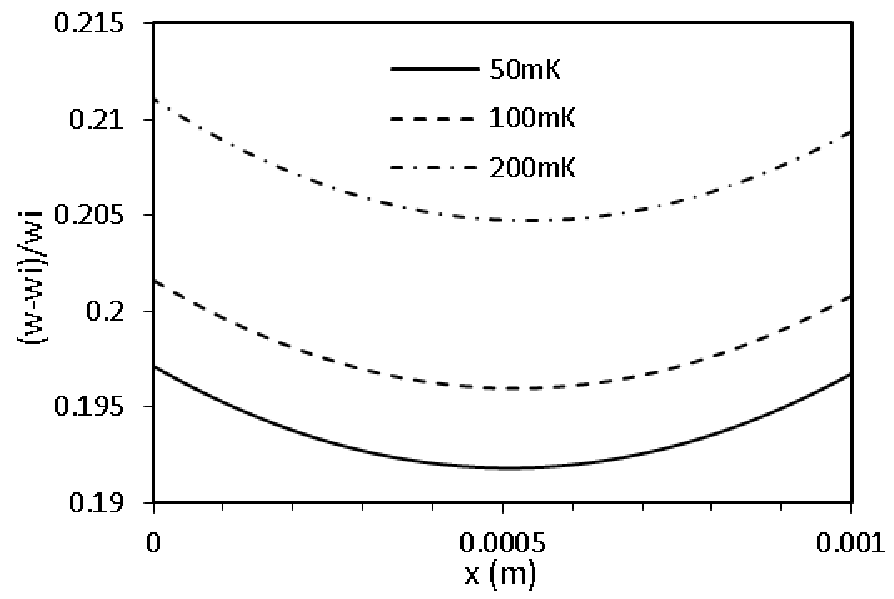

(b)
651

\subsection{Effect of initial mass fraction}

Fig. 10. Influence of the piston effect on the mass fraction perturbation without gravity with (a) $d \mathrm{LnK} 2 / \mathrm{dp} \neq 0$ and (b) $\mathrm{dLnK} 2 / \mathrm{dp}=0$ in Eq. (10) for $\mathrm{T}_{\mathrm{i}}=308.15 \mathrm{~K}$ and $\mathrm{t}=500 \mathrm{~s}$.

This explanation is confirmed by Fig. 10 where a case without the pressure term in Eq. (10) was tested. We can then observe that with the sole presence of the temperature term, a stronger heating increases the mass fraction in the entire volume (Fig. 10(b)). Consequently, the pressure term plays a major role in the expression of the adsorption rate $K_{a}$ (Eq. (10)) near the critical point. The evolution of the mass fraction profiles as a function of heating depicted in Fig. 10(a) is then directly attributable to the piston effect which is responsible for the strong and homogeneous increase of the pressure in the cavity. 
Table 4

The effect of the initial mass fraction for $\mathrm{T}_{\mathrm{i}}=308.15 \mathrm{~K}, \Delta \mathrm{T}=100 \mathrm{mK}$ and $\Delta \mathrm{T}=50 \mathrm{mK}$ at $\mathrm{t}=30 \mathrm{~s}$ and without gravity

\begin{tabular}{|c|c|c|c|}
\hline \multicolumn{4}{|c|}{$\Delta T=100 \mathrm{mK}$} \\
\hline $\mathbf{w}_{\mathbf{i}}$ & $\mathbf{w}-\mathbf{w}_{\mathbf{i}}(\mathbf{x}=\mathbf{0})$ & $w-w_{i}(x=H)$ & $\left(\mathbf{w}_{\mathrm{x}=0}-\mathbf{w}_{\mathrm{x}=\mathrm{H}}\right) / \mathbf{w}_{\mathrm{x}=0}$ \\
\hline $7.6751 \times 10^{-3}$ & $3.93 \times 10^{-4}$ & $3.88 \times 10^{-4}$ & $1.48 \times 10^{-2}$ \\
\hline $4.22 \times 10^{-3}$ & $1.65 \times 10^{-4}$ & $1.62 \times 10^{-4}$ & $1.97 \times 10^{-2}$ \\
\hline $2.11 \times 10^{-3}$ & $7.16 \times 10^{-5}$ & $6.99 \times 10^{-5}$ & $2.28 \times 10^{-2}$ \\
\hline $9.35 \times 10^{-4}$ & $2.95 \times 10^{-5}$ & $2.87 \times 10^{-5}$ & $2.46 \times 10^{-2}$ \\
\hline $7.6751 \times 10^{-4}$ & $2.39 \times 10^{-5}$ & $2.34 \times 10^{-5}$ & $2.49 \times 10^{-2}$ \\
\hline \multicolumn{4}{|c|}{$\Delta \mathrm{T}=50 \mathrm{mK}$} \\
\hline $\mathbf{w}_{\mathbf{i}}$ & $\mathrm{w}-\mathrm{w}_{\mathrm{i}}(\mathrm{x}=\mathbf{0})$ & $\mathbf{w}-\mathbf{w}_{\mathbf{i}}(\mathbf{x}=\mathbf{H})$ & $\left(\mathbf{w}_{\mathrm{X}=0}-\mathbf{w}_{\mathrm{X}=\mathrm{H}}\right) / \mathbf{w}_{\mathrm{X}=0}$ \\
\hline $7.6751 \times 10^{-3}$ & $3.94 \times 10^{-4}$ & $3.91 \times 10^{-4}$ & $7.44 \times 10^{-3}$ \\
\hline $4.22 \times 10^{-3}$ & $1.65 \times 10^{-4}$ & $1.64 \times 10^{-4}$ & $9.88 \times 10^{-3}$ \\
\hline $2.11 \times 10^{-3}$ & $7.15 \times 10^{-5}$ & $7.07 \times 10^{-5}$ & $1.15 \times 10^{-2}$ \\
\hline $9.35 \times 10^{-4}$ & $2.94 \times 10^{-5}$ & $2.91 \times 10^{-5}$ & $1.24 \times 10^{-2}$ \\
\hline $7.6751 \times 10^{-4}$ & $2.39 \times 10^{-5}$ & $2.36 \times 10^{-5}$ & $1.25 \times 10^{-2}$ \\
\hline
\end{tabular}

667

668

669

670

671 reported in Tables 4 and 5 for cases with and without gravity and for two temperature

672 increases $\Delta \mathrm{T}=50 \mathrm{mK}$ and $100 \mathrm{mK}$. The maximum value of $\mathrm{w}_{\mathrm{i}}$ corresponds to the solubility of

673 Naphthalene in $\mathrm{CO}_{2}$. We note that the difference between the left and right sides observed in

674 Fig. 8 without gravity can be quantified for each initial mass fraction and for the two

675 temperature increase. Then, the effect of the gravity is confirmed in Table 5 where

676 equilibrium is established between the two sides and only a slight difference can be observed

677 between the heated and the isothermal plates. A detailed analysis of Tables 4 and 5 reveals 


\subsection{Proximity of the LCEP}

694

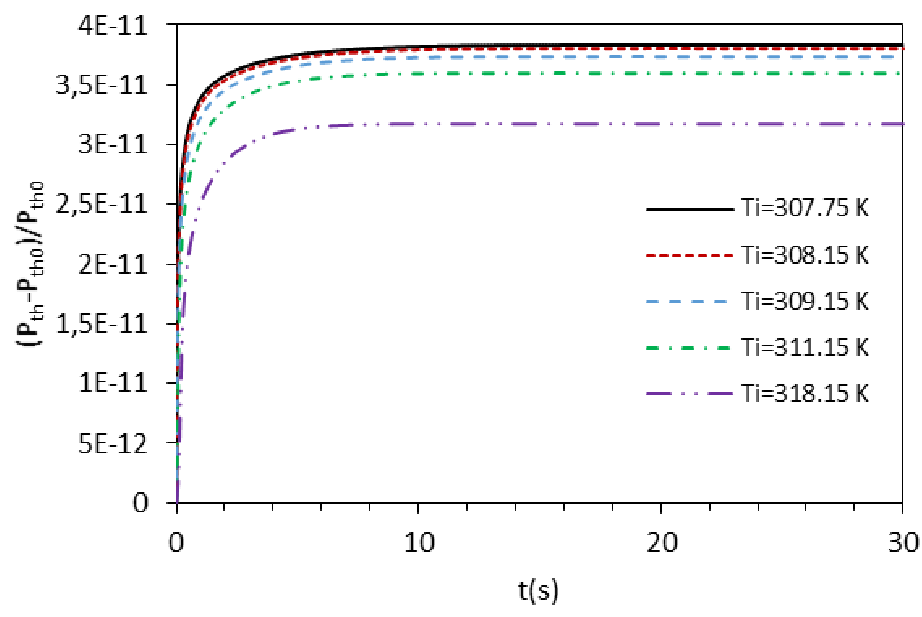

(a)

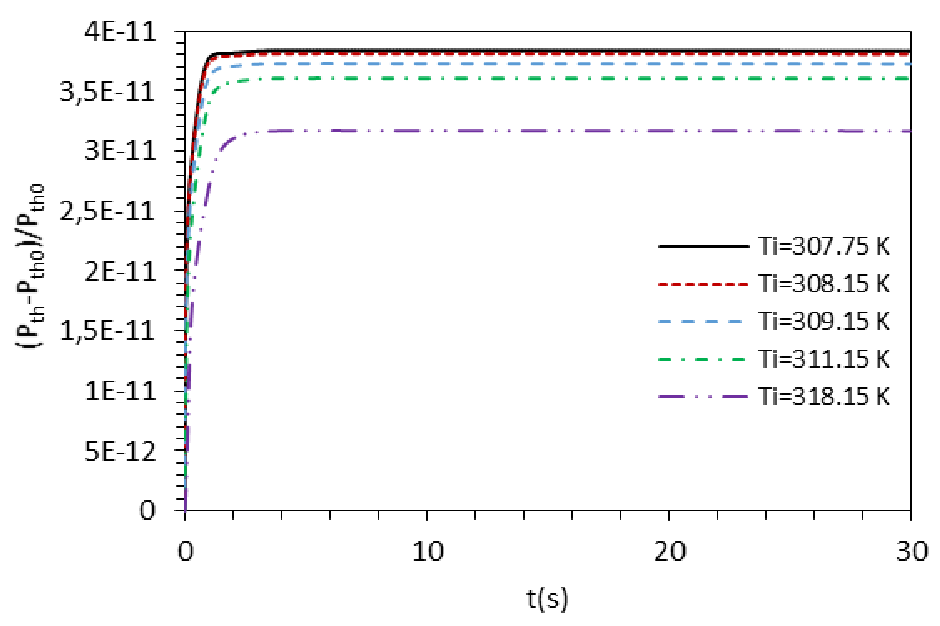

(b) 

corresponding to the solubility of Naphthalene in $\mathrm{CO}_{2}$ at temperature $\mathrm{T}_{\mathrm{i}}$ and density $\rho_{\mathrm{i}}$. The values of $\mathrm{w}_{\mathrm{i}}$ and the initial pressure $\mathrm{P}_{\text {thi }}$ calculated using the Peng-Robinson equation are reported in Table 6.

Table 6

Initial mass fraction $\mathrm{w}_{\mathrm{i}}$ and pressure $\mathrm{P}_{\text {thi }}$

\begin{tabular}{llll}
\hline $\mathbf{T}_{\mathbf{i}}(\mathbf{K})$ & $\boldsymbol{\rho}_{\mathbf{i}}\left(\mathbf{k g} \cdot \mathbf{m}^{-3}\right)$ & $\mathbf{w i}$ & $\mathbf{P}_{\text {thi }}(\mathbf{M P a})$ \\
\hline 307.75 & 470 & $7.6751 \times 10^{-3}$ & 8.67158154 \\
308.15 & 470 & $4.22 \times 10^{-3}$ & 8.73970242 \\
309.15 & 470 & $2.11 \times 10^{-3}$ & 8.90942413 \\
311.15 & 470 & $9.35 \times 10^{-4}$ & 9.22114740 \\
318.15 & 470 & $7.6751 \times 10^{-4}$ & 10.3949472 \\
\hline
\end{tabular}

701

702

703

704

705

706

707

708

709

710

711

712

713

714

715

A Similar behavior to that described in section 3.1 was observed for the mass fraction for different initial temperatures $T_{i}$ and temperature increases $\Delta T$. However, when we move away from the critical point, the partial derivatives of the equilibrium constant $\mathrm{K}_{2}$ with respect to temperature and pressure decrease (Figs. 2 and 3) and thus, the mass fraction at the heated and isothermal sides are influenced. Moreover, it must be noted that, since the initial mass fraction $\mathrm{w}_{\mathrm{i}}$ is fixed to the value corresponding to the solubility at $T_{\mathrm{i}}$ and $\rho_{\mathrm{i}}$, the resulting pressure gets higher as the initial temperature moves away from the critical one. As a consequence, the initial pressure belongs to the high pressure range where the derivative of $\mathrm{K}_{2}$ with respect to pressure is smaller. For all the initial temperatures $\left(\mathrm{T}_{\mathrm{i}}=307.75 \mathrm{~K}\right.$ to $\left.\mathrm{T}_{\mathrm{i}}=318.15 \mathrm{~K}\right)$, the piston effect generated by the boundary heating induces a fast and strong pressure rise in the entire volume before the pressure reaches a steady value. When the system is subjected to the Earth's gravity, convection accelerates the pressure increase (Fig. 11(b)). Yet, with or without gravity, above the critical point the piston effect becomes less effective and the pressure plateau for a given $\Delta \mathrm{T}$ gradually decreases. For $\mathrm{T}_{\mathrm{i}}=318.15 \mathrm{~K}$, steady value is much smaller 
showing that far enough from the critical point the previously observed effects on temperature and mass fraction will be reduced.

The effect on temperature and density can actually be observed in Figs. 12 and 13 comparing the instantaneous temperature and density fields near and far from the critical point (for $\mathrm{T}_{\mathrm{i}}=307.75 \mathrm{~K}$ and $\mathrm{T}_{\mathrm{i}}=318.15 \mathrm{~K}$ ). For these two initial temperatures, the characteristic time scales of the piston effect are $t_{\mathrm{PE}}=0.19 \mathrm{~s}$ and $\mathrm{t}_{\mathrm{PE}}=1.26 \mathrm{~s}$ respectively. On the other hand, the characteristic time of thermal diffusion is $t_{d}=115.7 \mathrm{~s}$ for $T_{i}=307.75 \mathrm{~K}$ and $t_{d}=49.23 \mathrm{~s}$ for $\mathrm{T}_{\mathrm{i}}=318.15 \mathrm{~K}$. Therefore, far from the critical point, the piston effect decreases in favor of the thermal diffusion as clearly shown by the temperature field in Fig. 12(b). The boundary layers become thicker and the thermal plumes are larger (Figs 12(b) and 13(b)). The figures also show that, near the critical point $\left(\mathrm{T}_{\mathrm{i}}=307.75 \mathrm{~K}\right)$, top and bottom plumes reach the opposite plate while the reduction of the piston effect away from the critical point reduces this phenomenon.

The lessening of the piston effect depicted by Fig. 11 is confirmed by Tables 7 and 8 which show that the pressure value corresponding to the equilibrium state after $t=30$ s decrease when moving away from the critical point for both temperature increases of $50 \mathrm{mK}$ and $100 \mathrm{mK}$ and for the two cases with and without gravity effect. It can be also noted that the gravity has a very little influence on the pressure evolution, since very close values are obtained for the pressure with and without gravity. Tables 7 and 8 also show how the proximity to the critical point affects the adsorbed amount at the two reactive walls. In the two cases, with or without gravity, a change in the variation of the mass fraction as a function of initial temperature can be observed for the farest value of $T_{i}$. Indeed, the mass fraction at the heated and isothermal plates regularly decreases when the system moves away from the critical temperature up to $\mathrm{T}_{\mathrm{i}}=311.15 \mathrm{~K}$. Then, a strong increase of the mass fraction is observed at $T_{i}=318.15 \mathrm{~K}$. A similar behavior change at the highest temperature can also be noted on the relative gap of 
mass fraction between the two sides. For example, for $\Delta \mathrm{T}=100 \mathrm{mK}$, the difference between the (Eq. (10)).

Table 7

The effect of the proximity to the critical temperature for $\Delta \mathrm{T}=100 \mathrm{mK}$ and $\Delta \mathrm{T}=50 \mathrm{mK}$ at $\mathrm{t}=30 \mathrm{~s}$ and without gravity

\begin{tabular}{|c|c|c|c|c|}
\hline & & $100 \mathrm{mK}$ & 8 & \\
\hline $\mathbf{T}_{\mathbf{i}}(\mathbf{K})$ & $w-w_{i}(x=0)$ & $\mathrm{w}-\mathrm{w}_{\mathrm{i}}(\mathrm{x}=\mathbf{H})$ & $\left(w_{x=0}-w_{x=H}\right) / w_{x=0}$ & $\left(\mathbf{P}_{\text {th }}-\mathbf{P}_{\text {th } 0}\right) / \mathbf{P}_{\text {th } 0}$ \\
\hline 307.75 & $4.09 \times 10^{-4}$ & $4.03 \times 10^{-4}$ & $1.3885 \times 10^{-2}$ & $3.8347 \times 10^{-11}$ \\
\hline 308.15 & $3.93 \times 10^{-4}$ & $3.88 \times 10^{-4}$ & $1.4824 \times 10^{-2}$ & $3.8053 \times 10^{-11}$ \\
\hline 309.15 & $3.75 \times 10^{-4}$ & $3.69 \times 10^{-4}$ & $1.6061 \times 10^{-2}$ & $3.7326 \times 10^{-11}$ \\
\hline 311.15 & $3.71 \times 10^{-4}$ & $3.65 \times 10^{-4}$ & $1.6067 \times 10^{-2}$ & $3.6028 \times 10^{-11}$ \\
\hline 318.15 & $4.77 \times 10^{-4}$ & $4.72 \times 10^{-4}$ & $1.0726 \times 10^{-2}$ & $3.1747 \times 10^{-11}$ \\
\hline \multicolumn{5}{|c|}{$50 \mathrm{mK}$} \\
\hline $\mathbf{T}_{\mathbf{i}}(\mathbf{K})$ & $\mathbf{w}-\mathbf{w}_{\mathbf{i}}(\mathbf{x}=\mathbf{0})$ & $w-w_{i}(x=H)$ & $\left(w_{x=0}-w_{x=H}\right) / w_{x=0}$ & $\left(\mathbf{P}_{\text {th }}-\mathbf{P}_{\text {th } 0}\right) / \mathbf{P}_{\text {th } 0}$ \\
\hline 307.75 & $4.10 \times 10^{-4}$ & $4.07 \times 10^{-4}$ & $6.9607 \times 10^{-3}$ & $1.9197 \times 10^{-11}$ \\
\hline 308.15 & $3.94 \times 10^{-4}$ & $3.91 \times 10^{-4}$ & $7.4364 \times 10^{-3}$ & $1.9046 \times 10^{-11}$ \\
\hline 309.15 & $3.75 \times 10^{-4}$ & $3.72 \times 10^{-4}$ & $8.0633 \times 10^{-3}$ & $1.8675 \times 10^{-11}$ \\
\hline 311.15 & $3.70 \times 10^{-4}$ & $3.67 \times 10^{-4}$ & $8.0684 \times 10^{-3}$ & $1.8016 \times 10^{-11}$ \\
\hline 318.15 & $4.76 \times 10^{-4}$ & $4.73 \times 10^{-4}$ & $5.3793 \times 10^{-3}$ & $1.5852 \times 10^{-11}$ \\
\hline
\end{tabular}

Table 8

The effect of the proximity to the critical temperature $\Delta \mathrm{T}=100 \mathrm{mK}$ and $\Delta \mathrm{T}=50 \mathrm{mK}$ at $\mathrm{t}=30 \mathrm{~s}$ and with gravity

\begin{tabular}{|c|c|c|c|c|}
\hline \multicolumn{5}{|c|}{$100 \mathrm{mK}$} \\
\hline $\mathbf{T}_{\mathbf{i}}(\mathbf{K})$ & $\mathrm{w}-\mathrm{w}_{\mathrm{i}}(\mathbf{x}=\mathbf{0})$ & $w-w_{i}(x=H)$ & $\left(w_{x=0}-w_{x=H}\right) / w_{x=0}$ & $\left(\mathbf{P}_{\text {th }}-\mathbf{P}_{\text {th } 0}\right) / \mathbf{P}_{\text {th } 0}$ \\
\hline 307.75 & $3.94 \times 10^{-4}$ & $3.93 \times 10^{-4}$ & $2.6531 \times 10^{-3}$ & $3.8326 \times 10^{-11}$ \\
\hline 308.15 & $3.78 \times 10^{-4}$ & $3.77 \times 10^{-4}$ & $2.7778 \times 10^{-3}$ & $3.8036 \times 10^{-11}$ \\
\hline 309.15 & $3.58 \times 10^{-4}$ & $3.57 \times 10^{-4}$ & $2.9648 \times 10^{-3}$ & $3.7315 \times 10^{-11}$ \\
\hline 311.15 & $3.53 \times 10^{-4}$ & $3.52 \times 10^{-4}$ & $3.0537 \times 10^{-3}$ & $3.6024 \times 10^{-11}$ \\
\hline 318.15 & $4.56 \times 10^{-4}$ & $4.55 \times 10^{-4}$ & $2.3868 \times 10^{-3}$ & $3.1752 \times 10^{-11}$ \\
\hline \multicolumn{5}{|c|}{$50 \mathrm{mK}$} \\
\hline $\mathbf{T}_{\mathbf{i}}(\mathbf{K})$ & $\mathrm{w}-\mathrm{w}_{\mathrm{i}}(\mathrm{x}=\mathbf{0})$ & $w-w_{i}(x=H)$ & $\left(w_{x=0}-w_{x=H}\right) / w_{x=0}$ & $\left(\mathbf{P}_{\text {th }}-\mathbf{P}_{\text {th } 0}\right) / \mathbf{P}_{\text {th } 0}$ \\
\hline 307.75 & $3.98 \times 10^{-4}$ & $3.97 \times 10^{-4}$ & $1.7511 \times 10^{-3}$ & $1.9199 \times 10^{-11}$ \\
\hline 308.15 & $3.82 \times 10^{-4}$ & $3.81 \times 10^{-4}$ & $1.8280 \times 10^{-3}$ & $1.9049 \times 10^{-11}$ \\
\hline 309.15 & $3.62 \times 10^{-4}$ & $3.61 \times 10^{-4}$ & $1.9335 \times 10^{-3}$ & $1.8679 \times 10^{-11}$ \\
\hline 311.15 & $3.56 \times 10^{-4}$ & $3.55 \times 10^{-4}$ & $1.9657 \times 10^{-3}$ & $1.8020 \times 10^{-11}$ \\
\hline 318.15 & $4.59 \times 10^{-4}$ & $4.58 \times 10^{-4}$ & $1.5165 \times 10^{-3}$ & $1.5860 \times 10^{-11}$ \\
\hline
\end{tabular}

751 


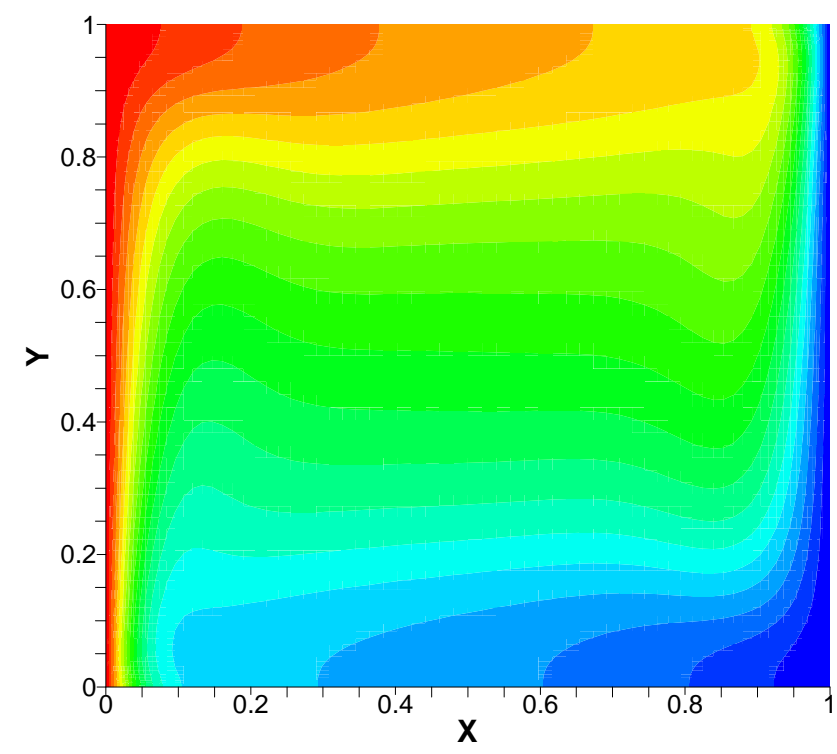

753

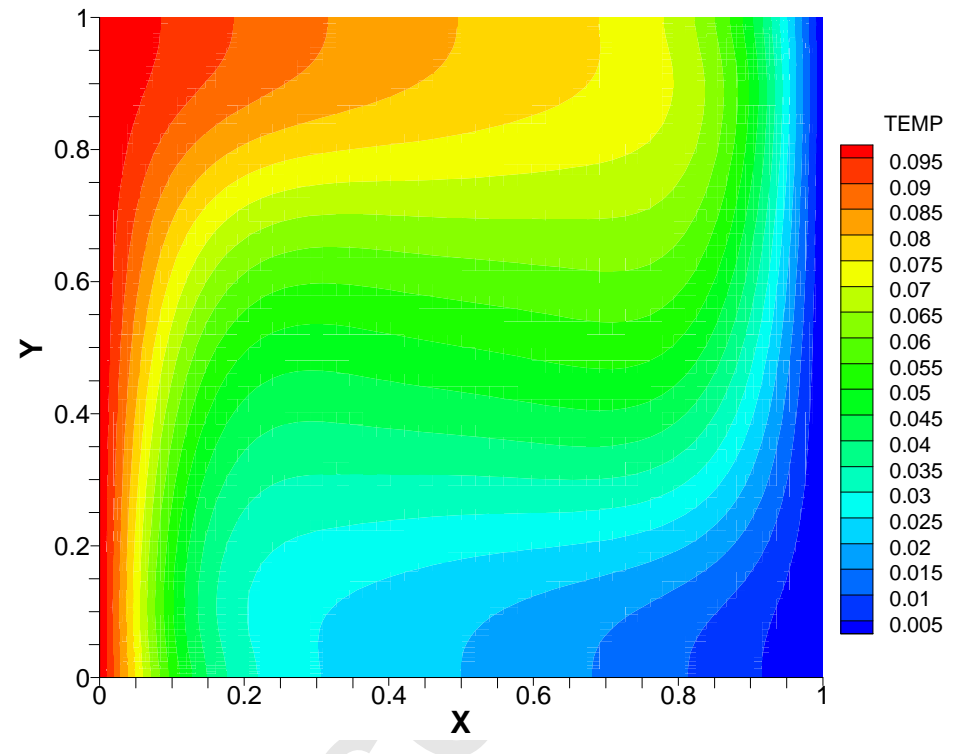

(b)

754 Fig. 12. Isosurfaces of temperature with gravity for (a) $\mathrm{T}_{\mathrm{i}}=307.75 \mathrm{~K}$ and (b) $318.15 \mathrm{~K}$ for $\Delta \mathrm{T}=100 \mathrm{mK}$ at $\mathrm{t}=500 \mathrm{~s}$.

755

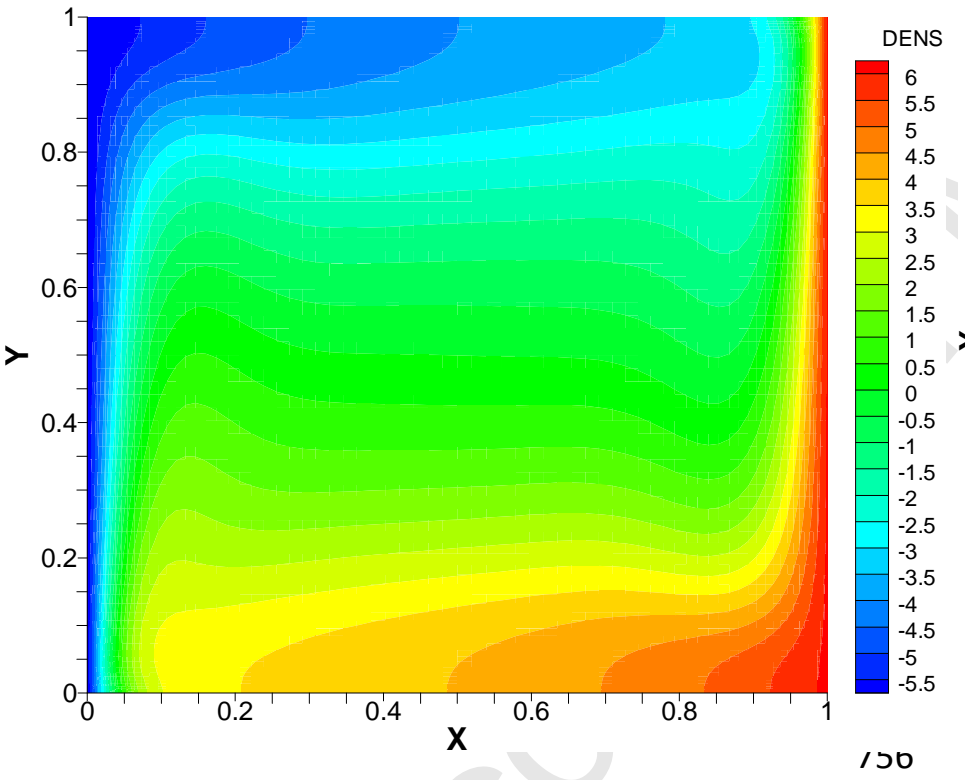

757

758 (a)

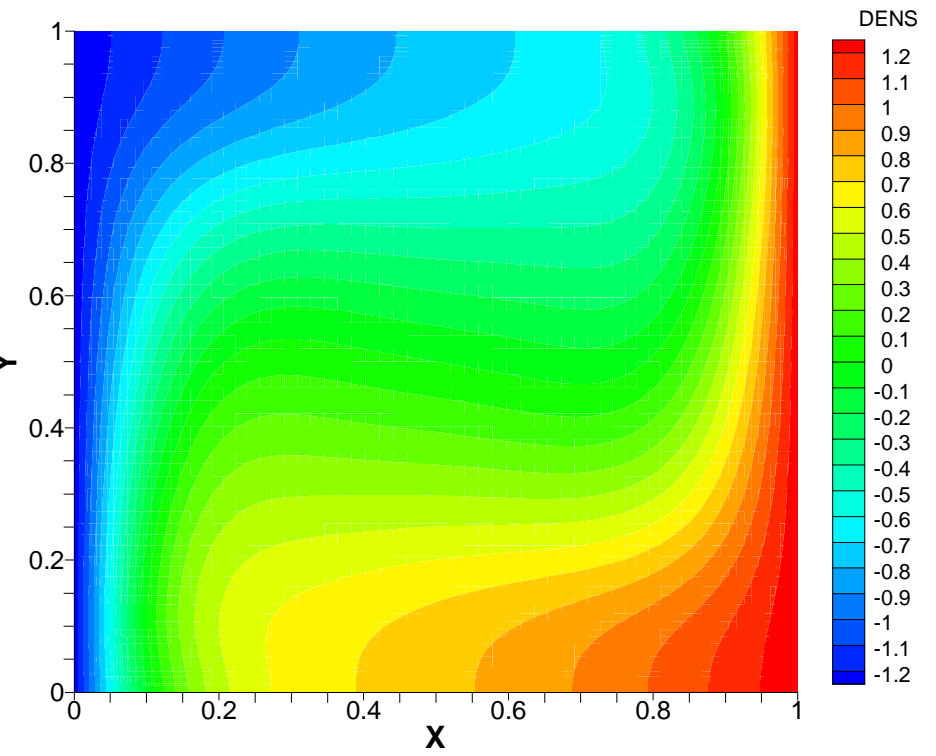

(b)

Fig. 13. Isosurfaces of density with gravity for (a) $\mathrm{T}_{\mathrm{i}}=307.75 \mathrm{~K}$ and (b) $318.15 \mathrm{~K}$ for $\Delta \mathrm{T}=100 \mathrm{mK}$ at $\mathrm{t}=500 \mathrm{~s}$ 


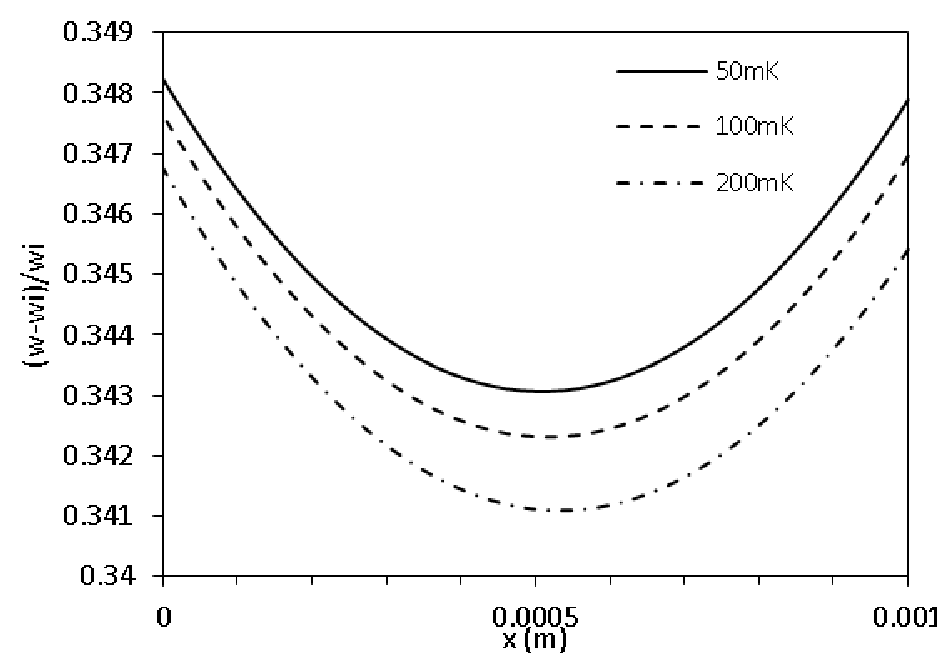

(a)

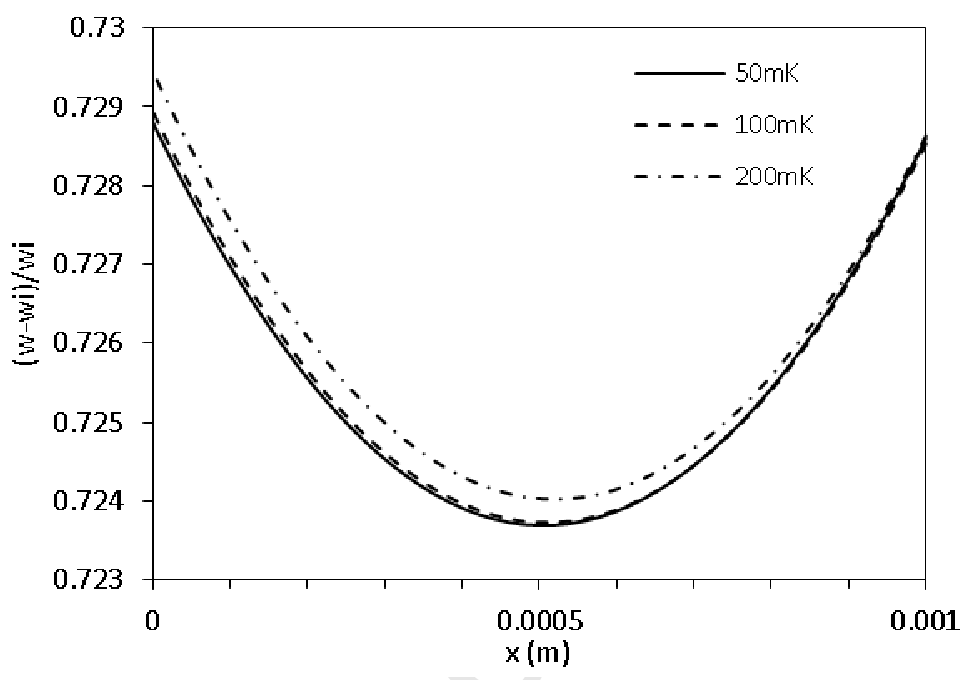

(b)

759 Fig. 14. Evolution of the mass fraction with the heating intensity for (a) $\mathrm{T}_{\mathrm{i}}=311.15 \mathrm{~K}$ at $\mathrm{t}=500 \mathrm{~s}$ and (b) $\mathrm{T}_{\mathrm{i}}=318.15 \mathrm{~K}$ at $\mathrm{t}=500 \mathrm{~s}$

760 The consequence of this decrease of the pressure term in Eq. (10) at the highest initial

761 temperature is depicted by Fig. 14 which shows the mass fraction variation between the two

762 plates for three temperature increases $(\Delta T=50,100$ and $200 \mathrm{mK})$ and two initial temperatures

$763\left(\mathrm{~T}_{\mathrm{i}}=311.15 \mathrm{~K}\right.$ and $\left.318.15 \mathrm{~K}\right)$. Though the tendency for $\mathrm{T}_{\mathrm{i}}=311.15 \mathrm{~K}$ is similar to that shown in

764 Fig. 10 for $T_{i}=308.15 K$, the behavior of the mass fraction distribution for $T_{i}=318.15 \mathrm{~K}$ is

765

766

767 and causes the enhancement of mass fraction with heating increase.

reversed: a stronger heating of the left side increases the mass fraction. The effect is more remarkable at the heated side than at the isothermal one where the profiles of $\Delta \mathrm{T}=50 \mathrm{mK}$ and $100 \mathrm{mK}$ merge. This behavior change is due to the competition between the derivatives of the equilibrium constant $\mathrm{K}_{2}$ with respect to temperature and pressure. Far away from the critical point, the diverging behaviors of the isothermal compressibility and of the volume expansivity disappear leading to smaller variations of the derivatives $\left(\partial \ln K_{2} / \partial T\right)_{p}$ and $\left(\partial \ln K_{2} / \partial P\right)_{T}$ (see Appendix D). Moreover, for a given heating intensity, the pressure increase generated by the piston effect is much lower for the highest initial temperature. As a result, the negative pressure term in Eq. (10) becomes negligible and only the temperature effect is highlighted 


\subsection{Influence of the Damköhler number}

\section{Table 9}

The effect of the Damköhler number on the mass fraction perturbation for $\mathrm{T}_{\mathrm{i}}=308.15 \mathrm{~K}$ and $\Delta \mathrm{T}=100 \mathrm{mK}$

\begin{tabular}{llll}
\hline Da & $\mathbf{1 0}^{-4}$ & $\mathbf{1 0}^{-5}$ & $\mathbf{1 0}^{-12}$ \\
\hline $\mathbf{w}-\mathbf{w}_{\mathbf{i}}(\mathbf{e n} \mathbf{x}=\mathbf{H})$ & $3.88 \times 10^{-3}$ & $3.88 \times 10^{-4}$ & $2.37 \times 10^{-10}$ \\
$\mathbf{w}-\mathbf{w}_{\mathbf{i}}(\mathbf{e n} \mathbf{x}=\mathbf{0})$ & $3.94 \times 10^{-3}$ & $3.93 \times 10^{-4}$ & $2.33 \times 10^{-10}$ \\
\hline
\end{tabular}

The results presented up to now were obtained for a Damköhler number fixed to $10^{-5}$. With reference to literature studies, it has been found that the Damköhler number for the adsorption of Naphthalene and other solutes such as toluene or benzene can vary from $10^{-3}$ to $10^{-14}[40-44]$. The Damköhler number was estimated using the available data in each research work. For the sake of comparison, three values of the Damköhler number were tested. Similar behaviors to those reported in the previous sections were found for temperature, pressure and mass fraction distribution. Only the mass fraction variations at the heated left side and the isothermal right one are presented in Table 9. The same tendency was found with high mass fraction at the heated plate for all the Damköhler numbers. Whereas the Naphthalene mass fraction is found to be very much smaller for the smallest Damköhler number, increasing Da by a decade results in an increase by a decade of the mass fraction for the larger values of Da.

\section{Conclusion}

In this paper we have presented new results and a detailed analysis of adsorption in a model binary dilute mixture, the Naphthalene- $\mathrm{CO}_{2}$ mixture, very close to the critical point. The results of this study revealed that sufficiently close to the mixture critical point, the increase of the wall heating remarkably affects the adsorbed amount at the two reactive boundaries and the mass fraction inside the cavity. More precisely, the adsorbed amount, as the bulk mass fraction, is reduced by increasing the wall heating. This peculiar behavior is attributed to the Piston effect, coupled with the divergent character of the derivative of the 


\section{Appendix A} processes.

adsorption equilibrium constant with respect to pressure. Far enough from the critical point, the Piston effect weakens and a classical behavior is observed. Our results also showed that this retrograde adsorption is obtained with and without gravity. However, in the presence of gravity, convection induces large thermal plumes along the hot and cold boundaries and tends to reduce the temperature gradients near the two walls leading to more symmetric profiles of the mass fraction. Finally, the effect of the Damköhler number was studied. The same behavior was found for all the values considered.

All the results presented in this paper were obtained for the Naphthalene- $\mathrm{CO}_{2}$ model mixture. However, we believe that this study can be relevant for many dilute binary mixtures. Indeed, the phenomena observed are due to the divergence of the solvent transport properties (namely the isothermal compressibility and the thermal expansion coefficient) near the critical point leading to the appearance of the Piston effect and to the divergence of the solute thermodynamic properties (such as infinite dilution partial molar volume). And these divergent behaviors occur in a universal way for large classes of systems. Therefore, similar results should be obtained for all binary dilute mixtures involving a non-volatile solute near the solvent's critical point and this kind of dilute mixtures is relevant for many adsorption

Acknowledgments: The authors acknowledge the financial support from the CNES (Centre National d'Etudes Spatiales).

823 The ratio of the isobaric and isochoric specific heats for pure $\mathrm{CO}_{2}, \gamma$, and for mixture, $\gamma_{m}$, 824 are calculated from the equation of state as follow: 
$825 \quad \gamma=1+\frac{T_{i}}{C_{v} \rho_{i}^{2}}\left(\frac{\partial P}{\partial T}\right)_{\rho}^{2}\left(\frac{\partial \rho}{\partial P}\right)_{T}$

826 The derivatives are calculated using the Peng-Robinson equation for pure $\mathrm{CO}_{2}$ :

$827\left(\frac{\partial P}{\partial T}\right)_{\rho}=\frac{(R / M) \rho}{1-b \rho}-f(\rho) \frac{d a}{d T}$

828 with $f(\rho)=\frac{\rho^{2}}{1+2 b \rho-b^{2} \rho^{2}}$ and $\frac{d a}{d T}=-1.487422 \frac{(R / M) \beta}{\rho_{c}} \sqrt{\frac{T_{c}}{T}}\left[1+\beta\left(1-\sqrt{T / T_{c}}\right)\right]$

$829 \quad\left(\frac{\partial \rho}{\partial P}\right)_{T}=\frac{(1-b \rho)^{2}}{(R / M) T-a(T)(1-b \rho)^{2} \frac{d f}{d \rho}}$

830 with $\frac{d f}{d \rho}=\frac{2 \rho(1+b \rho)}{\left(1+2 b \rho-b^{2} \rho^{2}\right)^{2}}$

831 In Eqs. (A2) and (A3), $a(T)$ and $b$ are the coefficients of the Peng-Robinson equation of 832 state written for mass variable in dimensional form.

$a_{i}(T)=1.487422 \frac{\left(R / M_{i}\right) T_{c i}}{\rho_{c i}}\left[1+\beta_{i}\left(1-\sqrt{T / T_{c i}}\right)\right]^{2}$

833

$b_{i}=0.253076 \frac{1}{\rho_{c i}}$

for $i=1,2$

834 In a similar way, the capacity ratio of the mixture, $\gamma_{m}$ is calculated as follow:

835

$$
\gamma_{m}=1+\frac{T_{i}}{C_{v} \rho_{i}^{2}}\left(\frac{\partial P}{\partial T}\right)_{\rho, w}^{2}\left(\frac{\partial \rho}{\partial P}\right)_{T, w}
$$

836 where the derivatives are calculated using the Peng-Robinson equation of state for the 837 mixture:

$838\left(\frac{\partial P}{\partial T}\right)_{\rho, w}=\frac{\left(R / M_{1}\right) \rho \theta(w)}{1-b(w) \rho / \theta(w)}-f(\rho, w)\left(\frac{\partial a}{\partial T}\right)_{w}$

839 with $f(\rho, w)=\frac{\rho^{2}}{1+2 b(w) \rho / \theta(w)-b(w)^{2} \rho^{2} / \theta(w)^{2}}$

840 and $\left(\frac{\partial a}{\partial T}\right)_{w}=\frac{d a_{1}}{d T}(1-w)^{2}+2 \frac{d a_{12}}{d T} w(1-w)+\frac{d a_{2}}{d T} w^{2}$

841 in which the derivatives $d a_{i} / d T$ are calculated as described above for pure component. 
$\left(\frac{\partial \rho}{\partial P}\right)_{T, w}=\frac{(1-b(w) \rho / \theta(w))^{2}}{\left(R / M_{1}\right) T \theta(w)-a(T, w)(1-b(w) \rho / \theta(w))^{2}\left(\frac{\partial f}{\partial \rho}\right)_{w}}$

843 with $\left(\frac{\partial f}{\partial \rho}\right)_{w}=\frac{2 \rho(1+b(w) \rho / \theta(w))}{\left(1+2 b(w) \rho / \theta(w)-b(w)^{2} \rho^{2} / \theta(w)^{2}\right)^{2}}$

$844 \quad \underline{\text { Appendix B }}$

845 The difference of the partial molar internal energies of the two components is expressed by:

$\bar{U}_{2}(T, \vartheta, y)-\bar{U}_{2}(T, \vartheta, y)=H_{2}^{0}\left(T_{0}\right)-H_{1}^{0}\left(T_{0}\right)+C p_{2}^{0}-C p_{1}^{0}+\frac{1}{2 \sqrt{2} \bar{b}} C O F 1$

846

$\operatorname{Ln}\left(\frac{\vartheta+(1-\sqrt{2}) \bar{b}}{\vartheta+(1+\sqrt{2}) \bar{b}}\right)+\frac{\operatorname{COF} 2}{\vartheta^{2}+2 \vartheta \bar{b}-\bar{b}^{2}}$

847 with $H_{2}^{0}\left(T_{0}\right)$ and $H_{1}^{0}\left(T_{0}\right)$ the perfect gas enthalpy of the two components at $\mathrm{T}_{0}=298.15 \mathrm{~K}$

848 and $y$ the mole fraction of component 2, calculated from the mass fraction by the formula:

849

$y=\frac{\left(M_{1} / M_{2}\right) w}{\theta(w)}$

850

$C p_{2}^{0}$ and $C p_{1}^{0}$ are the isobaric heat capacities of components 2 and 1 respectively as perfect

851 gas and their difference is expressed by:

852

$C p_{2}^{0}-C p_{1}^{0}=\left(A_{2}-A_{1}\right)\left(T-T_{0}\right)+\frac{1}{2}\left(B_{2}-B_{1}\right)\left(T^{2}-T_{0}^{2}\right)+\frac{1}{3}\left(C_{2}-C_{1}\right)\left(T^{3}-T_{0}^{3}\right)$

$+\frac{1}{4}\left(D_{2}-D_{1}\right)\left(T^{4}-T_{0}^{4}\right)$

853 Finally:

854

$C O F 1=\left\{-\frac{1}{\bar{b}} \frac{d \bar{b}}{d y}\left[\bar{a}-T\left(\frac{\partial \bar{a}}{\partial T}\right)_{y}\right]+\left(\frac{\partial \bar{a}}{\partial y}\right)_{T}-T\left(\frac{\partial}{\partial y}\left(\frac{\partial \bar{a}}{\partial T}\right)_{y}\right)_{T}\right\}$

855

856

$\operatorname{COF} 2=\frac{1}{\bar{b}}\left[\bar{a}-T\left(\frac{\partial \bar{a}}{\partial T}\right)_{y}\right]\left[\bar{b}\left(\overline{V_{2}}-\overline{V_{1}}\right)+\vartheta \frac{d \bar{b}}{d y}\right]$ 


$$
\frac{d \bar{b}}{d y}=-2 \bar{b}_{1}(1-y)+2 \bar{b}_{12}(1-2 y)+2 \bar{b}_{2} y
$$

857

$$
\begin{aligned}
& \left(\frac{\partial \bar{a}}{\partial y}\right)_{T}=-2 \bar{a}_{1}(1-y)+2 \bar{a}_{12}(1-2 y)+2 \bar{a}_{2} y \\
& \left(\frac{\partial}{\partial y}\left(\frac{\partial \bar{a}}{\partial T}\right)_{y}\right)_{T}=-2 \frac{d \bar{a}_{1}}{d T}(1-y)+2 \frac{d \bar{a}_{12}}{d T}(1-2 y)+2 \frac{d \bar{a}_{2}}{d T} y
\end{aligned}
$$

858 where $\bar{a}, \bar{b}, \bar{a}_{i}, \bar{b}_{i}, \bar{a}_{12}$ and $\bar{b}_{12}$ are the coefficients of the Peng-Robinson equation of state 859 written in molar variables and they are defined by:

860

$$
\bar{a}(T, y)=\bar{a}_{1}(T)(1-y)^{2}+2 \bar{a}_{12}(T) y(1-y)+\bar{a}_{2}(T) y^{2}
$$

$\bar{b}(y)=\bar{b}_{1}(1-y)^{2}+2 \bar{b}_{12} y(1-y)+\bar{b}_{2} y^{2}$

861 with

862

$\bar{a}_{i}(T)=1.487422 \frac{R T_{c i}}{\left(\rho_{c i} / M_{i}\right)}\left[1+\beta_{i}\left(1-\sqrt{T / T_{c i}}\right)\right]^{2}$

$\bar{b}_{i}=0.253076 \frac{1}{\left(\rho_{c i} / M_{i}\right)}$

$\bar{a}_{12}(T)=\sqrt{\bar{a}_{1}(T) \bar{a}_{2}(T)}\left(1-k_{12}\right)$

$\bar{b}_{12}=\frac{1}{2}\left(\bar{b}_{1}+\bar{b}_{2}\right)\left(1-l_{12}\right)$

863 In the expression of $\mathrm{COF} 2$, the difference of volumes of Naphthalene and $\mathrm{CO}_{2}$ is expressed 864 by:

865

$$
\bar{V}_{2}-\bar{V}_{1}=(1-2 y) \frac{\left[\frac{R T}{(\vartheta-\bar{b})^{2}}+\frac{2 \bar{a}(\vartheta-\bar{b})}{\left(\vartheta^{2}+2 \bar{b} \vartheta-\bar{b}^{2}\right)^{2}}\right] \frac{d \bar{b}}{d y}-\frac{\left(\frac{\partial \bar{a}}{\partial y}\right)_{T}}{\vartheta^{2}+2 \bar{b} \vartheta-\bar{b}^{2}}}{\frac{R T}{(\vartheta-\bar{b})^{2}}-\frac{2 \bar{a}(\vartheta+\bar{b})}{\left(\vartheta^{2}+2 \bar{b} \vartheta-\bar{b}^{2}\right)^{2}}}
$$

866 where $\vartheta$ is the molar volume of mixture.

867 Appendix C

868 In the expression of the velocity divergence $(\nabla . V)$ (Eq. (8)), the derivatives are calculated as 869 follow:

870

$$
\left(\frac{\partial F}{\partial T}\right)_{\rho, w}=\frac{\rho \theta(w)}{1-b^{*}(w) \rho / \theta(w)}-f^{*}(\rho, w)\left(\frac{\partial a^{*}}{\partial T}\right)_{w}
$$


871 with $f^{*}(\rho, w)=\frac{\rho^{2}}{1+2 b^{*}(w) \rho / \theta(w)-b^{*}(w)^{2} \rho^{2} / \theta(w)^{2}}$

$872 a^{*}$ and $b^{*}$ are those defined for Eq. (5) and are calculated using $a_{i}^{*}$ and $b_{i}^{*}$ in dimensionless 873 form (see section 2.2).

$874\left(\frac{\partial F}{\partial \rho}\right)_{T, w}=-\frac{T \theta(w)}{\left(1-b^{*}(w) \rho / \theta(w)\right)^{2}}+a^{*}(T, w)\left(\frac{\partial f}{\partial \rho}\right)_{w}$

875

876

$\left(\frac{\partial F}{\partial w}\right)_{\rho, T}=-\frac{\rho T}{\left(1-b^{*}(w) \rho / \theta(w)\right)^{2}}\left[\rho \frac{d b^{*}}{d w}+\left(1-2 b^{*}(w) \rho / \theta(w)\right)\left(\frac{M_{1}}{M_{2}}-1\right)\right]$

$+f(\rho, w)\left(\frac{\partial a^{*}}{\partial w}\right)_{T}-\frac{2 a^{*}(T, w) f(\rho, w)^{2}}{\rho \theta(w)} \times\left(1-b^{*}(w) \rho / \theta(w)\right) \times\left[\frac{d b^{*}}{d w}-\frac{b^{*}(w)\left(M_{1} / M_{2}-1\right)}{\theta(w)}\right]$

$877 \quad$ (C.3)

878

$879 \quad \underline{\text { Appendix D }}$

880 In Eqs. (11)-(12), the volume expansivity, $\alpha$, the isothermal compressibility, $\kappa$, the partial

881 molar volume, $\bar{\vartheta}_{2}^{m}$ and the partial molar residual enthalpy, $\bar{h}_{2}^{m}-h_{2}^{I G}$, are given by:

882

$\alpha=\frac{1}{\vartheta}\left(\frac{\partial \vartheta}{\partial T}\right)_{p}=\frac{\frac{\partial \bar{a} / \partial T}{\vartheta\left(\vartheta^{2}+2 \vartheta \bar{b}-\bar{b}^{2}\right)}-\frac{R}{\vartheta(\vartheta-\bar{b})}}{\frac{2 \bar{a}(\vartheta+\bar{b})}{\left(\vartheta_{i}^{2}+2 \vartheta \bar{b}-\bar{b}^{2}\right)^{2}}-\frac{R T}{(\vartheta-\bar{b})^{2}}}$

883

$\kappa=-\frac{1}{\vartheta}\left(\frac{\partial \vartheta}{\partial p}\right)_{T}$

884

$=\frac{1}{\left(-2 \bar{a}(\vartheta+\bar{b}) \vartheta /\left(\vartheta^{2}+2 \vartheta \bar{b}-\bar{b}^{2}\right)^{2}\right)+R T \vartheta /(\vartheta-\bar{b})^{2}}$

885

$\bar{\vartheta}_{2}^{m}=\kappa \vartheta\left[\frac{\vartheta-\bar{b}+B}{(\vartheta-\bar{b})^{2}}-\frac{\left(\vartheta^{2}+2 \vartheta \bar{b}-\bar{b}^{2}\right) A-2 \bar{a}(\vartheta-\bar{b}) B}{\left(\vartheta^{2}+2 \vartheta \bar{b}-\bar{b}^{2}\right)^{2}}\right]$

$A=2 \bar{a}_{12}, B=2 \bar{b}_{12}-\bar{b}_{1}$ 
886

$$
\bar{h}_{2}^{m}-h_{2}^{I G}=P_{t h} \bar{\vartheta}_{2}^{m}-R T+\frac{(T(\partial \bar{a} / \partial T)-\bar{a})\left(\vartheta B-\bar{b} \bar{\vartheta}_{2}^{m}\right)}{\bar{b}\left(\vartheta^{2}+2 \vartheta \bar{b}-\bar{b}^{2}\right)}
$$

$$
+\frac{1}{2 \sqrt{2} \bar{b}} \ln \left(\frac{\vartheta+(1-\sqrt{2}) \bar{b}}{\vartheta+(1+\sqrt{2}) \bar{b}}\right)\left[2 \frac{d \bar{a}_{12}}{d T} T-A-\frac{1}{\bar{b}}\left(T \frac{\partial \bar{a}}{\partial T}-\bar{a}\right) B\right]
$$

\section{References}

[1] C. Cagniard de la Tour, Exposé de quelques résultats obtenu par l'action combinée de la chaleur et de la compression sur certains liquides, tels que l'eau, l'alcool, l'éther sulfurique et l'essence de pétrole rectifiée, Annales de Chimie et de Physique. 21 (1822) 127-132.

[2] D. Aslanidou, C. Tsioptsias, C. Panayiotou, A novel approach for textile cleaning based on supercritical CO2 and Pickering emulsions, Journal of Supercritical Fluids. 76 (2013) 83-93.

[3] C. Ventosa, D. Rebiscoul, V. Perrut, V. Ivanova, O. Renault, G. Passemard, Copper cleaning in supercritical $\mathrm{CO}(2)$ for the microprocessor interconnects, Micro-electronic Engineering. 85 (2008) $1629-1638$.

[4] T. Ito, Y. Otani, H. Inomata, Performance of air filters cleaned by supercritical carbon dioxide, Separation and Purification Technology. 40 (2004) 41-46.

[5] M.P. Srinivasan, J.M. Smith, B.J. McCoy, Supercritical Fluid Desorption from Activated Carbon, Chemical Engineering Science. 45 (1990) 1885.

[6] G. Madras, C. Erkey, M. Orejuela, A. Akgerman, Supercritical Fluid Regeneration of Activated Carbon Loaded with Heavy Molecular Weight Organics, Industrial and Engineering Chemistry Research. 32 (1993) 1163.

[7] S. J. Macnaughton, N. R. Foster, Supercritical Adsorption and Desorption Behavior of DDT on Activated Carbon Using Carbon Dioxide. Industrial and Engineering Chemistry Research. 34 (1995) 275-282.

[8] C. S. Tan, D. C. Liou, Supercritical Regeneration of Activated Carbon Loaded with Benzene and Toluene. Industrial and Engineering Chemistry Research. 28 (1989a) 1222-1226.

[9] C. S. Tan, D. C. Liou, Desorption of Ethyl Acetate from Activated Carbon by Supercritical Carbon Dioxide. Industrial and Engineering Chemistry Research. 27 (1988) 988-991.

[10] A.T. Andrews, R.C. Ahlert, D.S. Kosson, Supercritical fluid extraction of aromatic contaminants from a sandy loam soil. Environnemental Progress and Sustainable Energy. 9 (1990) 204-210.

[11] C. Erkey, G. Madras, M. Orejuela, A. Akgerman, Supercritical Fluid Extraction of Heavy Molecular Weight Organic Contaminants from Soil, Environmental Science and Technology. 27 (1993) 1225.

[12] S. Kothandaraman, , R.C. Ahlert, E.S. Venkataramani, A.T. Andrews, Supercritical extraction of polynuclear aromatic hydrocarbons from soil. Environmental Progress. 11 (1992) 220-222.

[13] S.J. Macnanghton, N.R. Foster, I. Kikic, Proc. 3rd Int. Syrup. On Supercritical Fluids, Tome 2, pl,Strasbourg. France, 1994.

[14] R. Humayun, D.L. Tomasko, High-resolution adsorption isotherms of supercritical carbon dioxide on activated carbon, AIChE Journal. 46 (2000) 2065-2075. 
[15] L.G. Aranovich, D.M. Donohue, Adsorption isotherms for microporous adsorbents. Fuel Energy Abstracts. 37 (1996b) 231.

[16] C. H. Lochmüller, L.P. Mink, Adsorption isotherms on silica for methanol and 1-hexanol modifiers from supercritical carbon dioxide Journal of Chromatography A. 471: 357-366. DOI: 10.1016/S0021-9673(00)94183-9.

[17] G. Afrane, E. Chimowitz, Adsorption in near-critical binary solvent mixtures: thermodynamic analysis and data. Fluid Phase Equilibria, 111 (1995) 213-238.

[18] I. Raspo, S. Meradji, B. Zappoli, Heterogeneous reaction induced by the piston effect in supercritical binary mixtures. Chemical Engineering Science. 62 (2007) 4182-4192.

[19] I. Raspo, B. Zappoli, P. Bontoux, Fast Mass Transfer at a solid-supercritical fluid interface by Piston Effect. Proceedings of 4th ICCHMT May 17-20, Paris-Cachan (2005).

[20] U. Van Wasen, I. Swaid, G. M. Schneider, Physicochemical Principles and Applications of Supercritical Fluid Chromatography (SFC), Angewandte Chemie, Int. Ed. 19 (1980) 575

[21] C. R. Yonker, R. W. Wright, S. L. Frye, R. D. Smith, Mechanism of Solute Retention in Supercritical Fluid Chromatography, Supercritical Fluids: Chemical and Engineering Principles and Applications. Squires and Paulaitis, eds., ACS Symposium. 329 (1987) 14.

[22] J. J. Shim, K. P. Johnston, Adjustable Solute Distribution Between Polymers and Supercritical Fluids, AIChE Journal. 35 (1989) 1097.

[23] F. P. Schmitz, D. Leyendecker, E. Klesper, Chromatography with Mobile Phases in the Liquid and the Supercritical State, Berichte der Bunsengesellschaft physikalische. 88 (1984) 912.

[24] E. Klesper, F. P. Schmitz, Gradient Methods in Supercritical Fluid Chromatography, Supercritical Fluids. 1 (1988) 45.

[25] S. Paolucci, On the filtering of sound from the Navier-Stokes equations. Technical report, Sandia National Laboratories USA, SAND82-8257 (1982).

[26] Y. Arai, T. Sako, Y. Takebayashi, Supercritical fluids: Molecular Interactions, Physical Properties and new Applications. Springer, Berlin 2002.

[27] H. Higashi, Y. Iwai, Y. Takahashi, H. Uchida, Y. Arai, Diffusion coefficients of naphthalene and dimethylnaphthalene in supercritical carbon dioxide. Fluid Phase Equilibria. 144 (1998) 269-278.

[28] C. Nicolas, E. Neau, S. Meradji, I. Raspo, The Sanchez-Lacombe Lattice fluid model for the modeling of solids in supercritical fluids. Fluid Phase Euilibria. 232 (2005) 219-229.

[29] J. Ouazzani, Y. Garrabos, A new numerical algorithm for low Mach number supercritical fluids, Retrieved october 2007. (available at https://hal.archives-ouvertes.fr/hal-00142988).

[30] I. Raspo, J. Ouazzani, Un algorithme faible nombre de Mach pour la simulation des écoulements de fluides supercritiques par des méthodes spectrales, Proceedings of the 19th Congrès Français de Mécanique, C Rey, P. Bontoux, A. Chrysochoos Eds., ISSN 2103_6225, paper 337-S05 (2009).

[31] S. K. Jha, G. Madras, Modeling of adsorption equilibria in supercritical fluids, Journal of Supercritical Fluids. 32 (2004) 161-166.

[32] R.F. Chang, G. Morrison, J.M.H. Levelt Sengers, The critical dilemma of dilute mixtures, Journal of Physical Chemistry. 88 (1984) 3389-3391.

[33] R.F. Chang, J.M.H. Levelt Sengers, Behavior of dilute mixtures near the solvent's critical point, Journal of Physical Chemistry. 90 (1986) 5921-5927. 
[34] J. M. Vanel, R. Peyret, P. Bontoux, A pseudospectral solution of vorticity-stream function equations using the influence matrix technique. In Morton, K. W., and Baines, M. J. (eds.), Numerical Methods for Fluid Dynamics II. (1986) 463-475.

[35] S. Hugues, A. Randriamampianina, An improved projection scheme applied to pseudospectral methods for the incompressible Navier-Stokes equations. International Journal for Numerical Methods in Fluids. 28 (1998) 501-521.

[36] P. Haldenwang, G. Labrosse, S. Abboudi, M. Deville, Chebyshev 3D spectral and 2D pseudospectral solvers for the Helmholtz equation. Journal of Computational Physics. 55 (1984) 115128.

[37] I. Raspo, S. Hugues, E. Serre, A. Randriamampianina, P. Bontoux, A spectral projection method for the simulation of complex three - dimensional rotating flows, Computers \& Fluids. 31 (2002) 745767.

[38] G. Accary, I. Raspo, P. Bontoux, B. Zappoli, An adaptation of the low Mach number approximation for supercritical fluid buoyant flows, Comptes Rendus Mecanique. 331 (2005) 397404.

[39] D. Ameur, I. Raspo, Numerical simulation of the Poiseuille-Rayleigh-Bénard instability for a supercritical fluid in a mini-channel, Computational Thermal Sciences. 5 (2013) 107-118.

[40] F. A. Aisien, A. Amenaghawon, A. Adinkwuye, Batch study, equilibrium and kinetics of adsorption of naphthalene using waste tyre rubber granules. Journal of Xenobiotics. 4 (2014) 2264.

[41] B. Cabal, C. Ania, J. Parra, J. Pis, Kinetics of naphthalene adsorption on an activated carbon : Comparison between aqueous and organic media. Chemosphere. 76 (2009) 433-438.

[42] C. Long, J. Lu, A. Li, D. Hu, F. Liu, Q. Zhang, Adsorption of naphthalene onto the carbon adsorbent from waste ion exchange resin : Equilibrium and kinetic characteristics. Journal of Hazardous Materials. 150 (2008) 656-661.

[43] S. Lucas, M. Calvo, C. Palencia, M. Cocero, Mathematical model of supercritical CO2 adsorption on activated carbon effect of operating conditions and adsorption scale-up. Journal of Supercritical Fluids. 32 (2004) 193-201.

[44] Q. Shi, A. Li, Z. Zhu, B. Liu, Adsorption of naphthalene onto a high-surface-area carbon from waste ion exchange resin. Journal of Environmental Sciences. 25 (2012) 188-94.

[45] B. Zappoli, S. Amiroudine, P. Carles, J. Ouazzani, Thermoacoustic and buoyancy-driven transport in a square side-heated cavity filled with near-critical fluid. Journal Fluid Mechanics. 316 (1996) 53-72.

[46] G. Accary, I. Raspo, P. Bontoux, B. Zappoli, Three-dimensional Rayleigh-Bénard instability in a supercritical fluid, Comptes Rendus Mecanique. 332 (2004) 209-216.

[47] H. Boukari, J. N. Shaumeyer, M. E. Briggs, R. W. Gammon, Critical speeding up in pure fluids. Physical Review A. 41 (1990) 2260-2263.

[48] A. Onuki, H. Hao, R. Ferrell, A. Fast adiabatic equilibration in a single- component fluid near the liquid-vapor critical point. Physical Review A. 41 (1990) 2256-2259.

[49] B. Zappoli, D. Bailly, Y. Garrabos, B. Le Neindre, P. Guenoun, D. Beysens, Anomalous heat transport by the piston effect in supercritical fluids under zero gravity. Physical Review A. 41 (1990) 2264-2267. 
1004 [50] I. P. Vadim, E. B. S. (Ed.), 10-15 September 2000. Simulation of naturel convection in a side1005 heated cavity with a near-critical fluid. No. 229-234. Proceedings of the First International Symposium 1006 on Microgravity Research \& Application in Physical Sciences \& Biotechnology.

1007 